\title{
Highly ionized gas in the local ISM: Some like it hot?
}

\author{
B. Y. Welsh ${ }^{1}$ and R. Lallement ${ }^{2}$ \\ 1 Experimental Astrophysics Group, Space Sciences Laboratory, UC Berkeley, Berkeley, CA 94720, USA \\ e-mail: bwelsh@ssl.berkeley.edu \\ 2 Service d'Aéronomie du CNRS, 91371 Verrières-le-Buisson, France
}

Received 23 December 2004 / Accepted 18 February 2005

\begin{abstract}
We present $H S T$-STIS medium-resolution spectra $\left(R \sim 6.5 \mathrm{~km} \mathrm{~s}^{-1}\right)$ of the ultraviolet interstellar absorption lines observed towards 4 early-type stars located within the local interstellar medium (ISM), with sight-line distances $<186 \mathrm{pc}$ in the general direction of the Loop I superbubble $\left(l=330^{\circ}, b=+18^{\circ}\right)$. These data have been supplemented with high resolution $\left(R \sim 3 \mathrm{~km} \mathrm{~s}^{-1}\right)$ visible absorption observations of the NaI D-lines towards these 4 stars. Our main discovery is the detection of highly ionized absorption components of CIV, SiIV and NV towards the two most distant targets, HD 127381 and HD 142256. These lines-of-sight are known to cross both the near and far neutral interface boundaries to the Loop I cavity, in addition to intersecting the fragmented shell of neutral and partially ionized gas that defines the boundary to the Local Bubble. However, the presently measured narrow line profile-widths and their measured absorption intensities are found to be incompatible with theoretical models that predict high ion absorption due to the presence of evaporating cloud conduction interfaces. We conclude that the formation of high ions in the local ISM is highly dependent on the location of the absorbing gas clouds with respect to nearby sources of both hot X-ray emitting gas and/or photo-ionization.

Our observations have also revealed at least 6 gas clouds with distances ranging from $5 \mathrm{pc}$ to $150 \mathrm{pc}$ along these sight-lines. We have detected a cloud of neutral and partially ionized gas with a velocity of $-15 \mathrm{~km} \mathrm{~s}^{-1}$ and a hydrogen column density of $\log N(\mathrm{HI}+\mathrm{HII}) \sim 19.3 \mathrm{~cm}^{-2}$ that is thought to define the boundary to the Local Bubble cavity at a distance of $\sim 90 \mathrm{pc}$ in this galactic direction. The far neutral boundary to the Loop I superbubble cavity is also detected at a distance of $150-180 \mathrm{pc}$ and is composed of two cold clouds moving at velocities close to $V_{\text {helio }} \sim 0 \mathrm{~km} \mathrm{~s}^{-1}$ possessing a combined hydrogen column density of $\log N(\mathrm{HI}+\mathrm{HII}) \gg 19.5 \mathrm{~cm}^{-2}$. In contrast, we have also detected three low density, warm and partially ionized diffuse clouds with average velocities of $\sim-10,-23$ and $-32 \mathrm{~km} \mathrm{~s}^{-1}$, that are all located within a distance of $\sim 150 \mathrm{pc}$. The cloud component at $V \sim-23 \mathrm{~km} \mathrm{~s}^{-1}$ may be associated with the very local "G-cloud" at a distance of $<5 \mathrm{pc}$, but we also provide evidence for its placement at a greater distance. The measured velocities of the majority of the gas clouds we have detected along all 4 sight-lines are consistent with an inflow of gas into the LB cavity from the direction of the Loop I superbubble. This gas is flowing through a region of fragmentation at a distance of $\sim 90 \mathrm{pc}$ that represents the interaction region between the Loop I and Local Bubble cavities.
\end{abstract}

Key words. ISM: bubbles - ISM: kinematics and dynamics

\section{Introduction}

Hot and highly ionized interstellar gas plays an important role in all evolutionary models of galaxies and clusters, such that obtaining a good understanding of its emission and interaction processes is of paramount importance. A useful tool in the study of the multi-phase structure of the interstellar medium, and in particular that of the hot gas component, is the local interstellar medium (LISM) observed at the $10-150 \mathrm{pc}$ scale, since it contains all three phases of the IS gas. However, the detailed physical characteristics of the plasma that fills the Local Bubble (LB) cavity, the 50-150 pc wide volume of very rarefied IS gas surrounding the Sun, are still a matter of great debate. Hot, one million $\mathrm{K}$ gas is widely believed to fill the LB cavity, as implied by the detection of the ubiquitous diffuse soft X-ray background emission (Snowden et al. 1998). Indeed, this volume of space has been found to be largely devoid of cold and dense neutral gas, as shown by maps of the 3-D spatial distribution of sodium (NaI) absorption within $250 \mathrm{pc}$ by Lallement et al. (2003). These maps show that the LB cavity is north-south elongated and open-ended, like a chimney extending into the overlying northern and southern inner galactic halo regions (Welsh et al. 1999; Crawford et al. 2002). The local cavity is also connected to surrounding "interstellar bubbles" through several narrow tunnels of low neutral gas density and its overall shape suggests that it is being "squeezed" by these adjacent younger bubbles which are over-pressured with respect the older and more quiescent LB. It should also be noted that there are also many concentrations of warm $(5000-10000 \mathrm{~K})$ and diffuse clouds that reside within the LB region, the most famous being the local clouds in the near vicinity of the Sun (Lallement et al. 1995). 
Table 1. Stellar target information.

\begin{tabular}{ccccccc}
\hline \hline Star & $(l, b)$ & $m_{v}$ & Sp & $E(B-V)$ & $\begin{array}{c}V \sin i \\
\left(\mathrm{~km} \mathrm{~s}^{-1}\right)\end{array}$ & $\begin{array}{c}\text { Hipparcos distance } \\
(\mathrm{pc})\end{array}$ \\
\hline HD 128345 (A1) & $\left(320.1^{\circ},+99^{\circ}\right)$ & 4.0 & B5V & 0.01 & 240 & $95(+7,-6)$ \\
HD 127381 (A2) & $\left(318.9^{\circ},+9.3^{\circ}\right)$ & 4.4 & B2II & 0.02 & 119 & $176(+36,-20)$ \\
HD 138769 (B1) & $\left(331.0^{\circ},+8.8^{\circ}\right)$ & 4.5 & B3IV & 0.02 & 106 & $133(+14,-12)$ \\
HD 142256 (B2) & $\left(334.0^{\circ},+7.0^{\circ}\right)$ & 7.0 & B8V & 0.03 & $>150^{*}$ & $186(+35,-26)$ \\
\hline
\end{tabular}

* Derived from UV stellar lines.

According to several theoretical models, conductive interfaces between ambient hot $\left(10^{6} \mathrm{~K}\right)$ gas and embedded cold/warm clouds should produce semi-hot $\left(10^{5} \mathrm{~K}\right)$ gas that contains high ions such as $\operatorname{CIV}(\lambda 1548 \AA), \operatorname{SiIV}(\lambda 1394 \AA)$ and OVI $(\lambda 1032 \AA$ ) (Slavin 1989; Borkowski et al. 1990). These interfaces should surround all of the diffuse gas clouds within the LB and, more importantly, the dense neutral boundary to the LB cavity itself should also be lined with similar transition layers. However, the search for this highly ionized gas within $100 \mathrm{pc}$ has thus far remained elusive. For example, absorption measurements by both HST GHRS and STIS towards nearby hot stars and white dwarfs have failed to detect significant levels of both CIV and SiIV ions for sight-lines distances <100 pc (Bertin et al. 1995; Holberg et al. 1999). In addition, recent FUSE observations of local hot white dwarf stars have also failed to detect significant levels of OVI absorption (which are indicative of $\sim 300000 \mathrm{~K}$ gas) to a limit which is a factor of 5 lower than that predicted by theory (Oegerle et al. 2005). We further note that the present detection upper limits for these high ion absorption lines in the LB are barely compatible with the value predicted by theory from just one conductive interface, although a recent model that requires the rather extreme configuration of tightly entangled and tangential magnetic fields completely enveloping all gas clouds can explain this apparent detection deficiency (Cox \& Helenius 2003). In contrast, all these aforementioned high ions have been routinely detected with strong absorption lines by both HST and FUSE for distances $>200$ pc i.e. beyond the neutral boundary to the LB.

The search for emission from local gas with temperatures in the 50000-500000 K regime has also been in vain. Shelton (2003) has failed to detect locally-generated OVI line-emission within the LB and the NASA CHIPS satellite's failure to detect local EUV line-emission is best explained by an extremely low emission measure for the putative hot LB gas that contradicts previously derived values from soft X-ray background data (Hurwitz et al. 2005). Furthermore, it has recently been shown that a significant fraction of the diffuse soft X-ray background emission is generated in the interplanetary medium as a result of the interaction between high ions in the solar wind and counterflowing interstellar neutrals (Cravens et al. 2001; Lallement 2004). This implies a lower pressure for hot gas in the LB by an amount between $15-50 \%$. However, this reduced hot gas pressure is still barely compatible with the observed intermediate ion column densities, and since conductive interfaces were the unique potential source of the ionization of helium in the local interstellar clouds their absence leaves their high ionization state (of $\sim 40 \%$ ) still unexplained (Wolff et al. 1999).

Clearly, there still remain many unanswered questions as to why the detection of hot and highly ionized gas residing within $200 \mathrm{pc}$ (if it exists at all) has been so difficult to achieve. The evidence seems favor these high ions (which are routinely detected towards more distant stars) originating at, or near, the interface of the LB with the surrounding denser gas of the general ISM. Therefore, we have proposed a program of ultraviolet absorption observations of the interstellar sight-lines towards 4 nearby stars using the HST STIS instrument to test this assumption. The 4 stellar targets were paired into two observational sets with similar interstellar sight-lines (pointing in the general direction of the Loop I superbubble) that pass through the LB cavity region. One stellar target in each sight-line pair was of a distance that placed it close to the boundary of the rarefied local cavity, while the other target in each pair was at a distance just beyond this nominal neutral boundary to the LB. In this Paper, we present the results of these absorption measurements that have revealed local high ion absorption profiles with unusually small doppler line-widths.

\section{Observations}

We have carried out ultraviolet absorption observations towards the four stars listed in Table 1, which lists their relevant astronomical data obtained from the Simbad on-line archive. We list a lower limit for the stellar rotational velocity of the star HD 142256, based on measurement of the FWHM of several stellar lines in its UV spectrum. For convenience we now refer to each of the two sight-line pair directions as "A" (towards $l \sim 320^{\circ}$ ) and "B" (towards $l \sim 330^{\circ}$ ) and the associated target stars as "A1" (HD 128345), "A2" (HD 127381), "B1" (HD 138769) and "B2" (HD 142256), where the suffixes " 1 " and " 2 " refer respectively to the near and far target of each sight-line pair.

The present absorption observations were made under the NASA HST Cycle 12 Guest Observer program GO-09876. These data were gained with the STIS instrument (Woodgate et al. 1998) through the $0.2 \times 0.05$ arcsec aperture (with neutral density filtering) using the E140M echelle grating, and the photons were collected by the far UV Multi-Anode Microchannel-Array (MAMA) detector. This configuration resulted in a spectral resolving power of $R \sim 46000\left(6.5 \mathrm{~km} \mathrm{~s}^{-1}\right)$ 
for all the spectral orders which were simultaneously recorded over the 1150-1690 ̊ range.

The data were processed using the CALSTIS data processing pipeline software that accounts for inter-order background (scattered-light) subtraction, flat-fielding, image linearity and wavelength calibration. The data have been doppler corrected to remove the effect of spacecraft motion, such that the output spectra are given in the helio-centric velocity scale. The accuracy of the STIS velocity scale has been found by other authors to be $\pm 1 \mathrm{~km} \mathrm{~s}^{-1}$ (Fox et al. 2003). We concur with this asessment, based on the similarity between our measured heliocentric velocities of the strongest NaI D-line absorption components observed at high resolution towards HD 127381 ( $V_{\text {helio }}=-0.6 \mathrm{~km} \mathrm{~s}^{-1}$ ) which are similar to the velocities presently determined for the neutral UV lines of CI and CII.

The exposure times for each target were such that a resul$\operatorname{tant} S / N$ ratio of $>30: 1$ was acheived for wavelengths in the 1250-1700 A region, with a decreasing ratio value for shorter wavelengths.

\section{Interstellar analysis}

We have determined the local stellar continua for the interstellar absorption lines listed in Table 2 for the four target stars using a multi-order polynomial fit. Errors associated with this continuum placement are automatically generated by the computer software routine and have been discussed in Welsh et al. (1990). In general, due to the high rotational velocity of the target stars, the local continua were well-behaved and the placement error was small for all the lines currently presented. The resultant residual intensity profiles for the major absorption lines of interest in this study are shown in Figs. 1 to 4 . A future publication will discuss all of the UV interstellar lines detected towards the 4 stars, focussing on a discussion of element abundances in the local gas.

The residual intensity absorption profiles were fit with multiple absorption components (identified with interstellar gas "clouds") using a line-fitting program described in Sfeir et al. (1999). This program assigns a 3-parameter theoretical fit to the observed absorption profiles by assigning values for the interstellar gas cloud component (helio-centric) velocity, $V$, a Gaussian velocity dispersion, $b$, and a cloud component ion column density, $N$. The fit parameters derived from this process are accurate for absorption lines that are not fully saturated, whereas large uncertainties can exist for values derived for the central cores of highly saturated spectral lines. Since all 4 sight-lines sample relatively low-density interstellar gas clouds that reside within $200 \mathrm{pc}$, in the majority of cases linecomponent saturation was not a problem and the best-fit values of $b$ and $N$ are shown in Table 2 for each spectral line detected towards all 4 targets. In cases where line-component saturation was evident, the derived best-fit parameter values have been marked with asterisks (**) in the table.

Our profile fits have required 6 or fewer absorption components with velocities that are self-consistent between each UV line species and are also consistent with our interstellar $\mathrm{NaI}$ absorption measurements along these 4 sightlines. We have also been guided in these fits by the high resolution $(R \sim 110000)$ observations of the interstellar CaII lines towards stars in this general direction by Crawford (1991, 2000, 2001). Due to the moderate spectral resolution of our UV data, the line-profile fits have been performed using the minimum number of absorption components and when more than one line of a particular species is available (such as the SII doublet), both profiles were fit simultaneously. We have used the following criterion of Vallerga et al. (1993) to determine if the data support the addition of extra absorption components (which will always improve the fit at some statistical level). If the absolute $\chi^{2}$ statistical error between the observed and computed model data points decreased by more than 11.1 , then the addition of another cloud component to fit the data was considered statistically significant.

Errors for the derived UV line component column densities are also listed in Table 2 and conservative upper limits to the column densities of absorption lines that were undetected in the data were calculated from multiplying the equivalent width of the largest noise feature located within $\pm 30 \mathrm{~km} \mathrm{~s}^{-1}$ of the rest wavelength of the line by a factor of 3 . The best-fit doppler widths (from which upper limits to the gas component temperature can be derived) are typically accurate to $\sim 30 \%$. All of our model fits to the absorption spectra are shown superposed on their observed UV line-profiles in Figs. 1-4.

In Fig. 5 we show the visible NaI D1 and D2 interstellar line-profiles recorded towards the 4 target stars. These data have been taken from high resolution $(R \sim 110000)$ observations using the coude echelle spectrograph at the European Southern Observatory (La Silla, Chile) in April 1998, as initially reported in Lallement et al. (2003). We also list the bestfit model parameters to these $\mathrm{NaI}$ absorption profiles in Table 2.

\section{Discussion}

The 4 target stars that comprise the two sight-line pairs ("A" and "B") all lie in the direction of a giant radio continuum feature called Loop I (or North Polar Spur) that is centered on $\left(l=329^{\circ}, b=+17.5^{\circ}\right)$ at a distance of $\sim 130$ pc (Heiles 1998). The radio emission intensity maximum traces almost a complete ring around this central point and has a diameter of $\sim 115^{\circ}$ (Berkhuijsen et al. 1971). This superbubble is thought to be produced by the collective stellar winds and several consecutive SN events originating within the Sco-Cen OB association and the associated interstellar gas is expanding and interacting with the neutral gas wall that surrounds our own LB cavity. The Loop I superbubble cavity itself is filled with hot gas at a temperature of $T \sim 10^{6.5} \mathrm{~K}$ and is an appreciable source of soft X-ray emission (Egger \& Aschenbach 1995). An expanding shell of HI gas has been found to surround Loop I, moving with a velocity of $\sim-12 \mathrm{~km} \mathrm{~s}^{-1}$ (de Geus 1992). High resolution $\mathrm{NaI}$ and CaII absorption measurements of many sight-lines in this galactic direction by both Crawford (1991, 2000) and Centurion \& Vladilo (1991) confirm the general outflow of gas away from the Sco-Cen OB association, with the majority of absorption components possessing negative velocities with respect to the local standard of rest. Unfortunately, little is known concerning the physical properties of the ionized interstellar gas that is present in the 


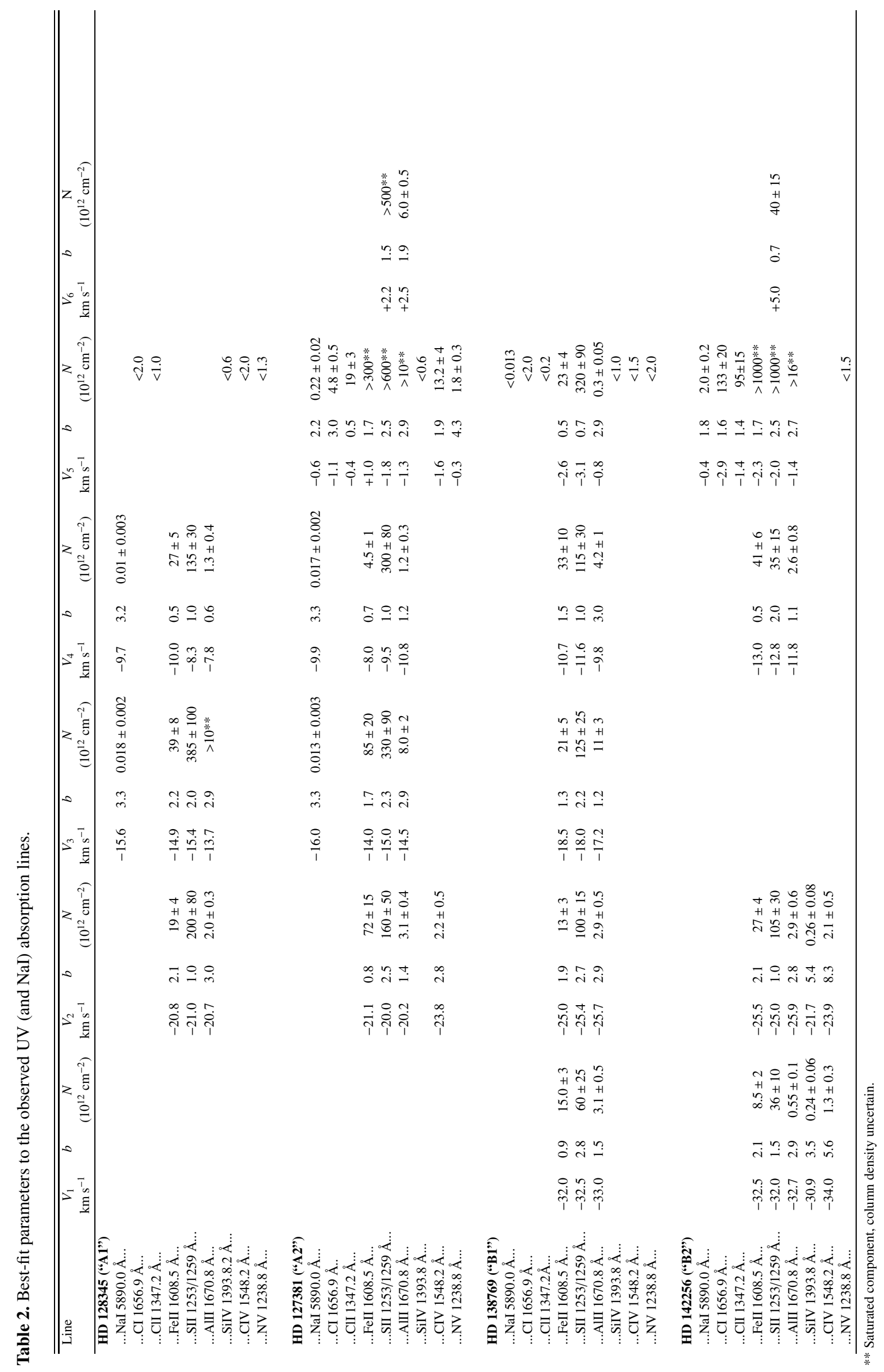


HD 128345

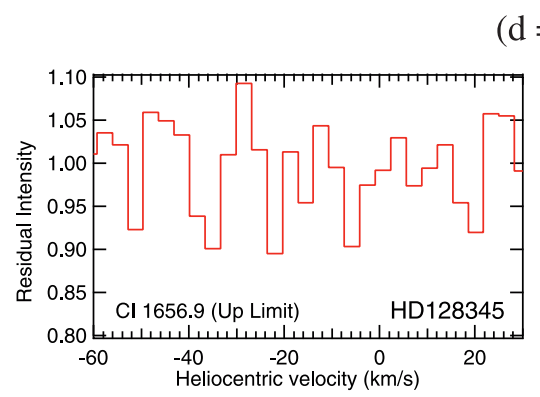

(d=95pc)
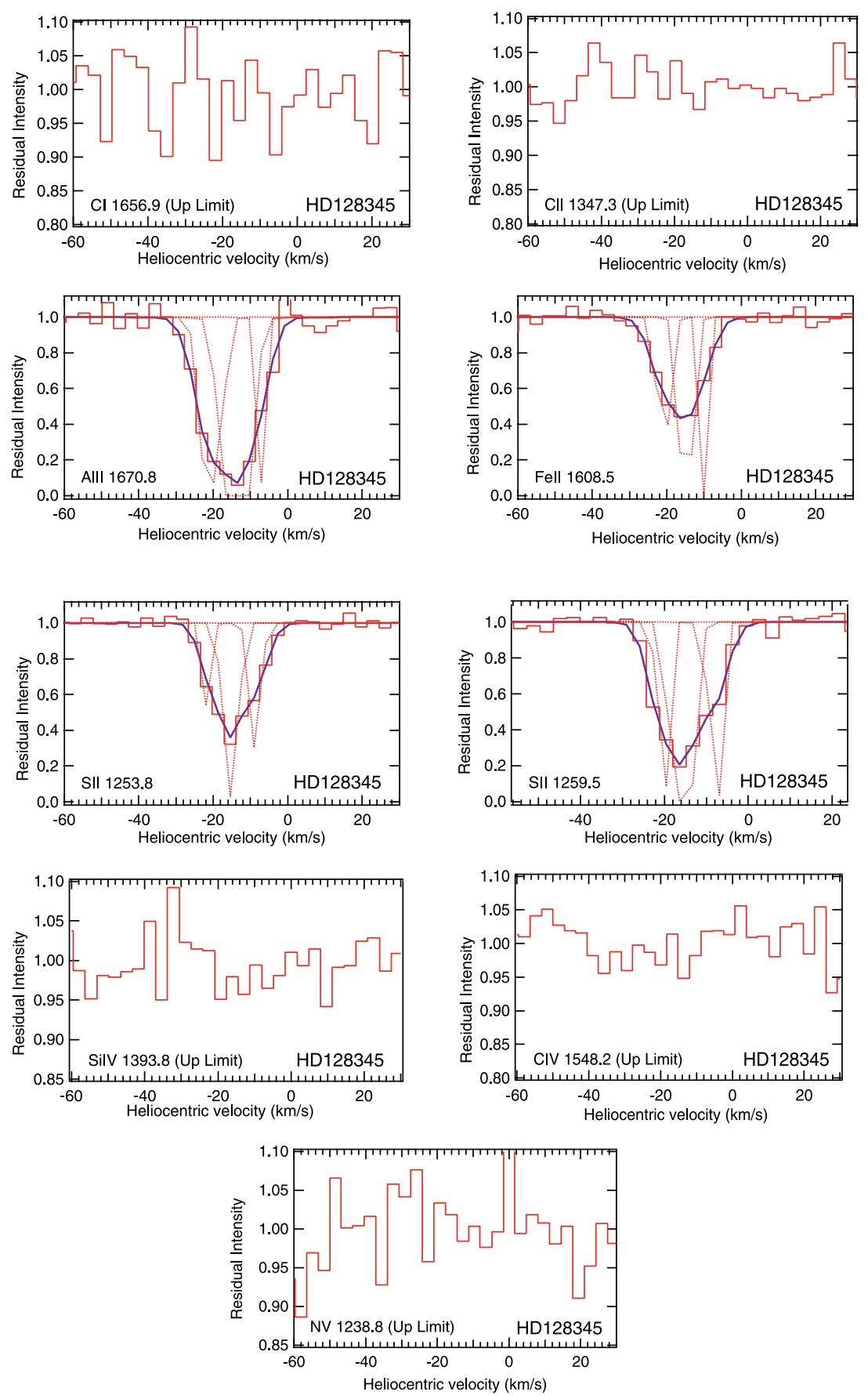

Fig. 1. Ultraviolet absorption line-profiles detected towards HD 128345 (sight-line "A1"). Solid thick lines represent the best-fit model to the observed data points, which are shown by the lighter lines. Actual model absorption components (unconvolved) are shown by dotted lines.

sight-line towards Loop I. However, our present STIS observations are ideally suited to reveal the absorption properties of the interstellar gas associated with such ionized regions. The absorption line profiles shown in Figs. 1-4 were specifically selected to demonstrate the range of ionization that may be present in the local interstellar gas through which the four sight-lines pass. Predominantly cold and neutral gas is sampled by the absorption lines of NaI $5890.0 \AA(<5.1 \mathrm{eV})$, CI $1656.9 \AA(<11.3 \mathrm{eV})$ and CII $1347.2 \AA(<13.0 \mathrm{eV})$, where the value in parenthesis is the ionization potential. The lines of FeII 1608.5 $\AA$ (7.9 to $16.2 \mathrm{eV}$ ), SII 1253.8/1259.5 $(10.4$ to $23.3 \mathrm{eV}$ ) and AlII $1670.8 \AA$ (5.9 to $18.8 \mathrm{eV}$ ) can be formed in warmer and more ionized regions, whereas absorption due to SiIV $1393.8 \AA$ (33.5 to $45.1 \mathrm{eV}$ ), CIV $1548.2 \AA$ (47.9 to $64.5 \mathrm{eV})$ and NV $1238.8 \AA$ (77.5 to $97.9 \mathrm{eV})$ is normally indicative of far hotter and highly ionized interstellar regions.

It is clear from the studies of Crawford $(1991,2000)$ that both the kinematics and the spatial distribution of the neutral 


\section{HD 127381}

$(\mathrm{d}=176 \mathrm{pc})$
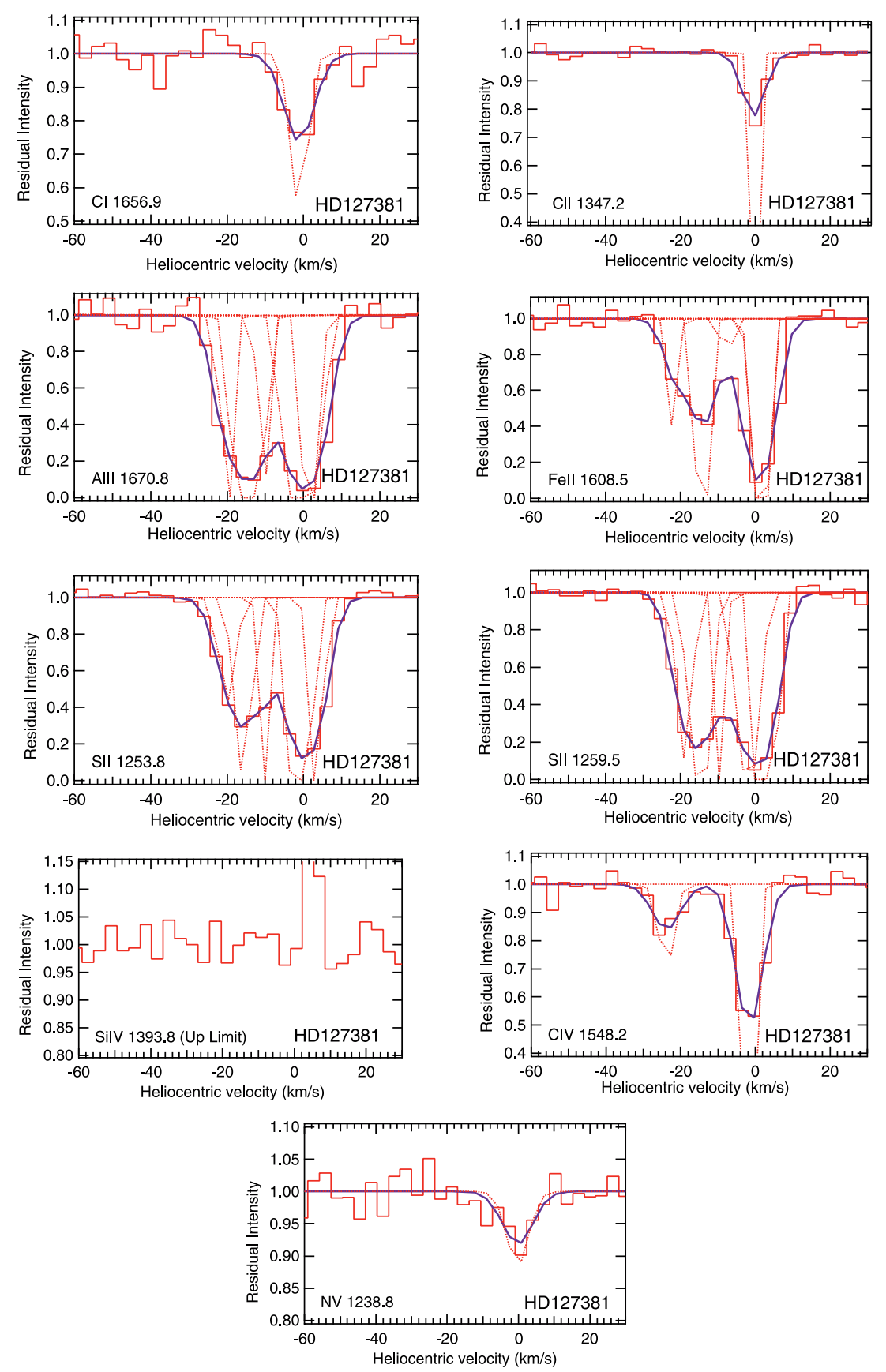

Fig. 2. Ultraviolet absorption line-profiles detected towards HD 127381 (sight-line "A2"). Solid thick lines represent the best-fit model to the observed data points, which are shown by the lighter lines. Actual model absorption components (unconvolved) are shown by dotted lines.

gas density are (unfortunately) quite complex in the directions we have selected to observe. de Geus (1992) has summarized the complexity of the interaction between the stellar winds of the Sco-Cen OB association and the ambient interstellar matter and has sub-divided the whole region into 3 main areas (the Upper Scorpius, the Upper Centaurus-Lupus and Lower Centaurus-Crux regions) as defined by the 3 large HI shell loops that appear to surround each stellar sub-group. The sight-lines to our 4 targets each pass through the Upper
Centaurus-Lupus region. In Fig. 6 we show the position of these 4 target sight-lines with respect to the distribution of neutral gas density as determined from the 2-D spatial density mapping method of NaI presented by Lallement et al. (2003). In addition, we also show the approximate distances to the six absorption components (i.e. interstellar gas-clouds) that we have detected along the " $\mathrm{A}$ " and "B" sight-lines which are discussed in the following sections. A summary of the physical properties of these clouds can be found in Table 3 . 
HD 138769

$(\mathrm{d}=133 \mathrm{pc})$
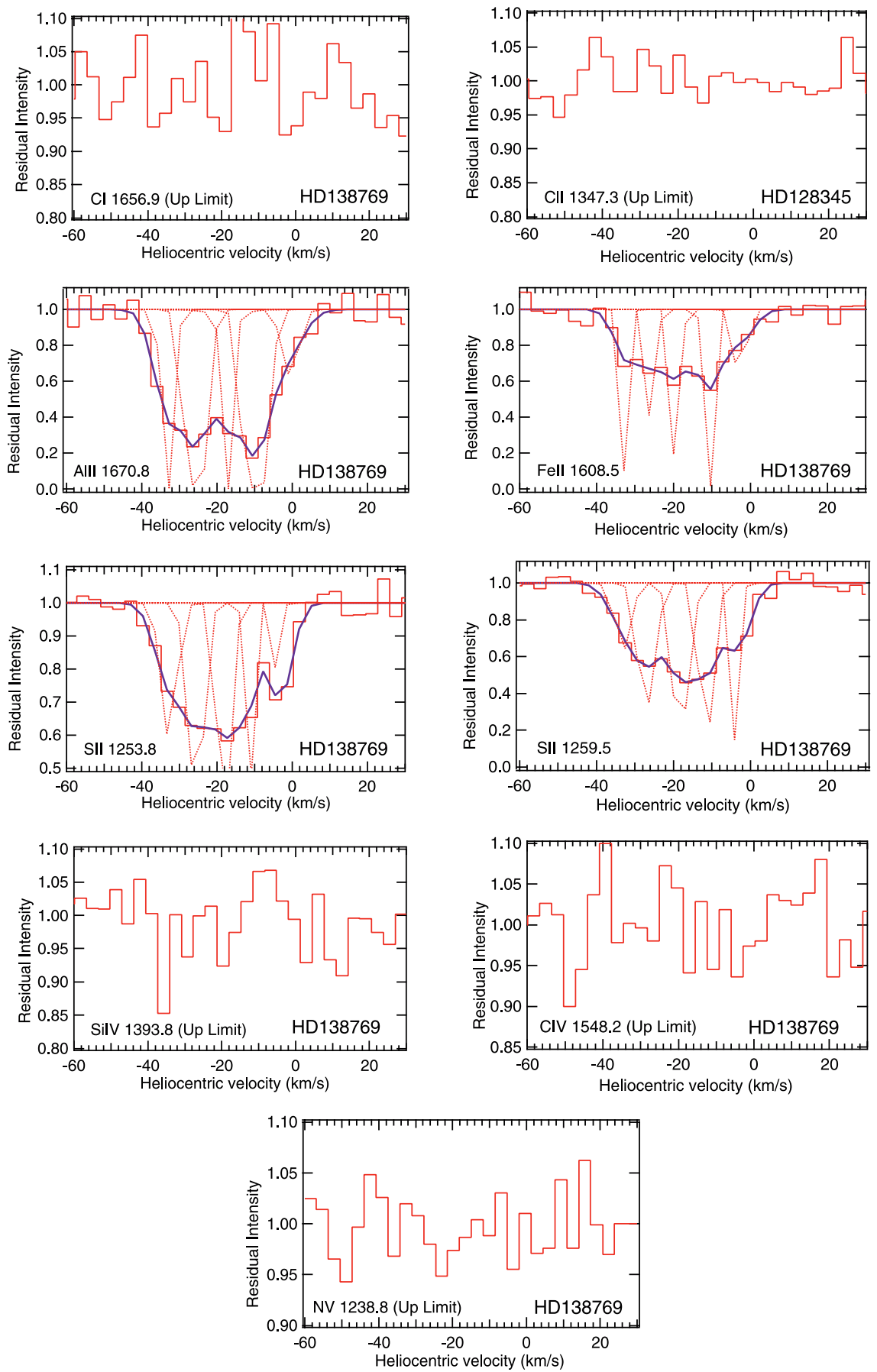

Fig. 3. Ultraviolet absorption line-profiles detected towards HD 138769 (sight-line "B1"). Solid thick lines represent the best-fit model to the observed data points, which are shown by the lighter lines. Actual model absorption components (unconvolved) are shown by dotted lines.

This new schematic map of the spatial density of cold gas within $250 \mathrm{pc}$ viewed close to the galactic plane has been constructed using $\mathrm{NaI}$ absorption data from other published target sight-lines that are contained within a restricted range of galactic latitude that is closer to that of our 4 target stars (i.e. $b=+10^{\circ}$ ). The reader should note that the average correlation length between each of the $\mathrm{NaI}$ absorption measurements towards the stellar targets that were used to construct this map is $\sim 25$ pc. Furthermore, taken together with a typical error of $\sim \pm 15 \mathrm{pc}$ in the Hipparcos distance to each of these targets, it is clear that less confidence can be placed on the fidelity of resultant cloud sizes and their distance placement for spatial scales $<30$ pc. However, it is clear from Fig. 6 that the sightlines towards all 4 stars pass through a "gap" in the neutral boundary to the local cavity at a distance of $\sim 75 \mathrm{pc}$. This gap appears to be a fragmentation in the dense wall of neutral gas 


\section{HD 142256}

$$
(\mathrm{d}=186 \mathrm{pc})
$$
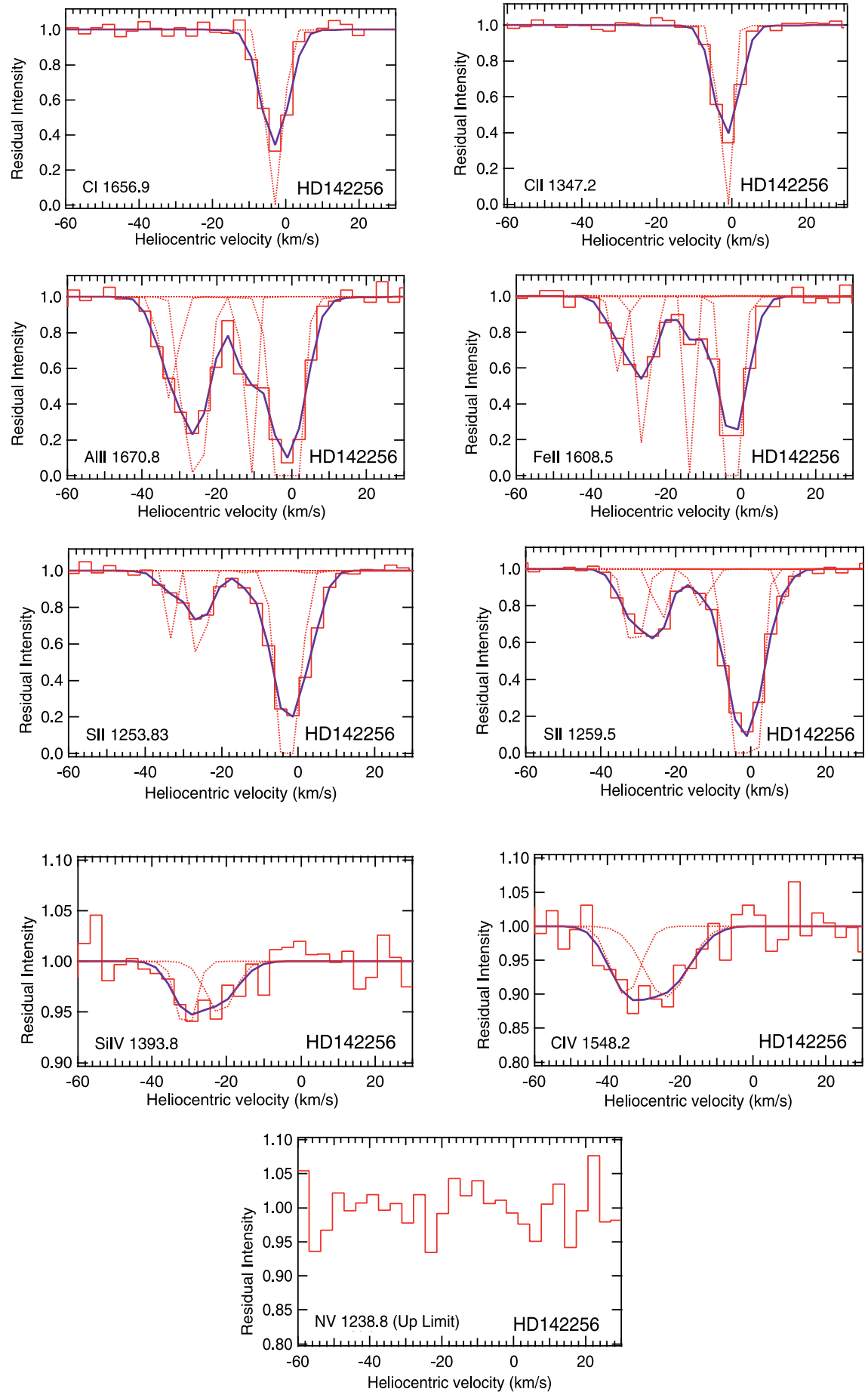

Fig. 4. Ultraviolet absorption line-profiles detected towards HD 142256 (sight-line "B2"). Solid thick lines represent the best-fit model to the observed data points, which are shown by the lighter lines. Actual model absorption components (unconvolved) are shown by dotted lines.

that separates the Loop I superbubble from our own local cavity. It would seem likely that this is the interaction region between the outflowing (ionized) gas from the Sco-Cen/Loop I region and the gas associated with the Local Bubble cavity and it surrounding boundary wall. Recently, Breitschwerdt et al. (2000) have proposed a model in which the diffuse low-density clouds that are known to exist within the local ISM region have their origins in the fragmentation of this interaction region. Our UV observations are well-suited to detect these local neutral and ionized cloud(lets), and we now procede to discuss the kinematic and physical state of these clouds observed in absorption along each of the 4 target sight-lines. 


\section{HD 128345}

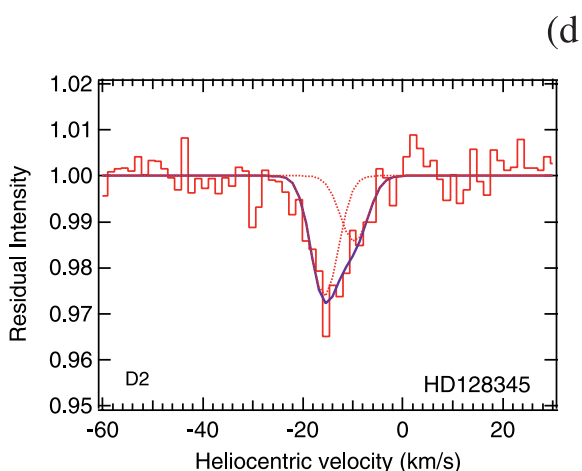

$$
(\mathrm{d}=95 \mathrm{pc})
$$

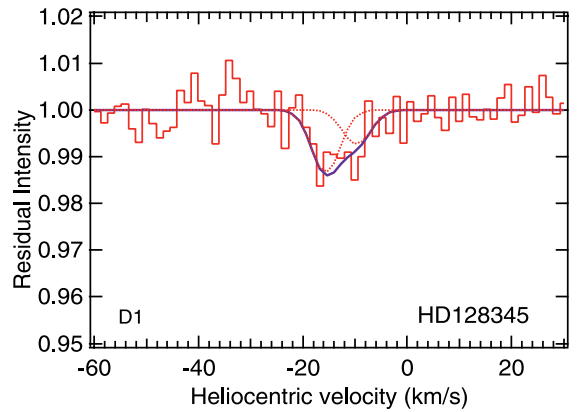

\section{HD 127381}

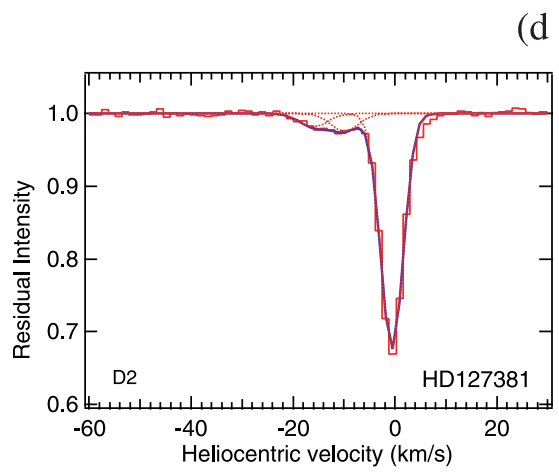

$(\mathrm{d}=176 \mathrm{pc})$

\section{HD 142256}

$(\mathrm{d}=186 \mathrm{pc})$
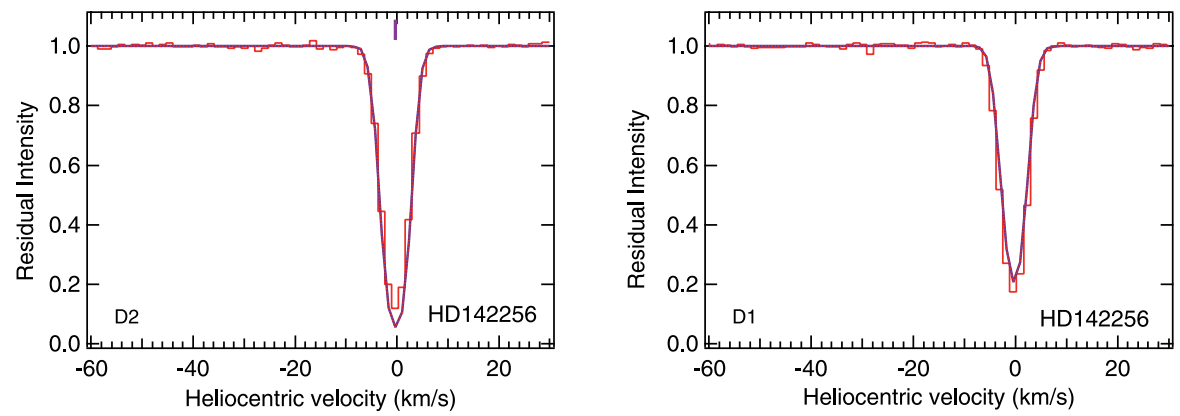

Fig. 5. NaI D2 and D1 interstellar absorption line-profiles towards HD 128345, HD 127381 and HD 142256 recorded at a spectral resolution of $R \sim 110000$. Solid thick-lines represent the best-fit model to the observed data points, which are shown by the lighter lines. Actual model absorption components (unconvolved) are shown by dotted lines.

\subsection{Interstellar absorption towards HD 128345 and $H D$ 127381: The neutral and low ionization gas along sight-line " $A$ "}

\section{The $V \sim-15 \mathrm{~km} \mathrm{~s}^{-1}$ cloud}

Our NaI observations towards "A1" $(d=95 \mathrm{pc})$ have revealed a weakly absorbing neutral cloud with a velocity of $V \sim-15.6 \mathrm{~km} \mathrm{~s}^{-1}$. Gas with this velocity has also been detected in NaI towards the angularly-close star HD 125238 $(d=108 \mathrm{pc})$ by Welsh et al. (1994). However, we note that neutral gas absorption at this velocity has not been detected in the UV lines of CI and CII towards "A1". If we assume the empirical relationship between the column densities of $\mathrm{NaI}$ and $\mathrm{CI}$ given by Jenkins \& Shaya (1979), then our presently measured low value of NaI column density of $\log N(\mathrm{NaI}) \sim 10.4 \mathrm{~cm}^{-2}$ for the $V \sim-15 \mathrm{~km} \mathrm{~s}^{-1}$ component would correspond to a column density for the CI $1656 \AA$ line that is below the present $H S T$-STIS detection threshold of $\log N(\mathrm{CI}) \sim 12.5 \mathrm{~cm}^{-2}$. No interstellar $\mathrm{NaI}$ has been detected towards any angular-close star with a distance $<83 \mathrm{pc}$ (HD 118991, Lallement et al. 2003), which therefore places a distance limit to this cloud of $\sim 90 \mathrm{pc}$. Absorption at a similar velocity is also revealed in the UV profiles of the FeII, SII and AlII lines towards "A1", which indicates that partially ionized and warmer gas is also present in this cloud in addition to the neutral gas revealed by the $\mathrm{NaI}$ observations. Gas with this velocity is also detected in the 


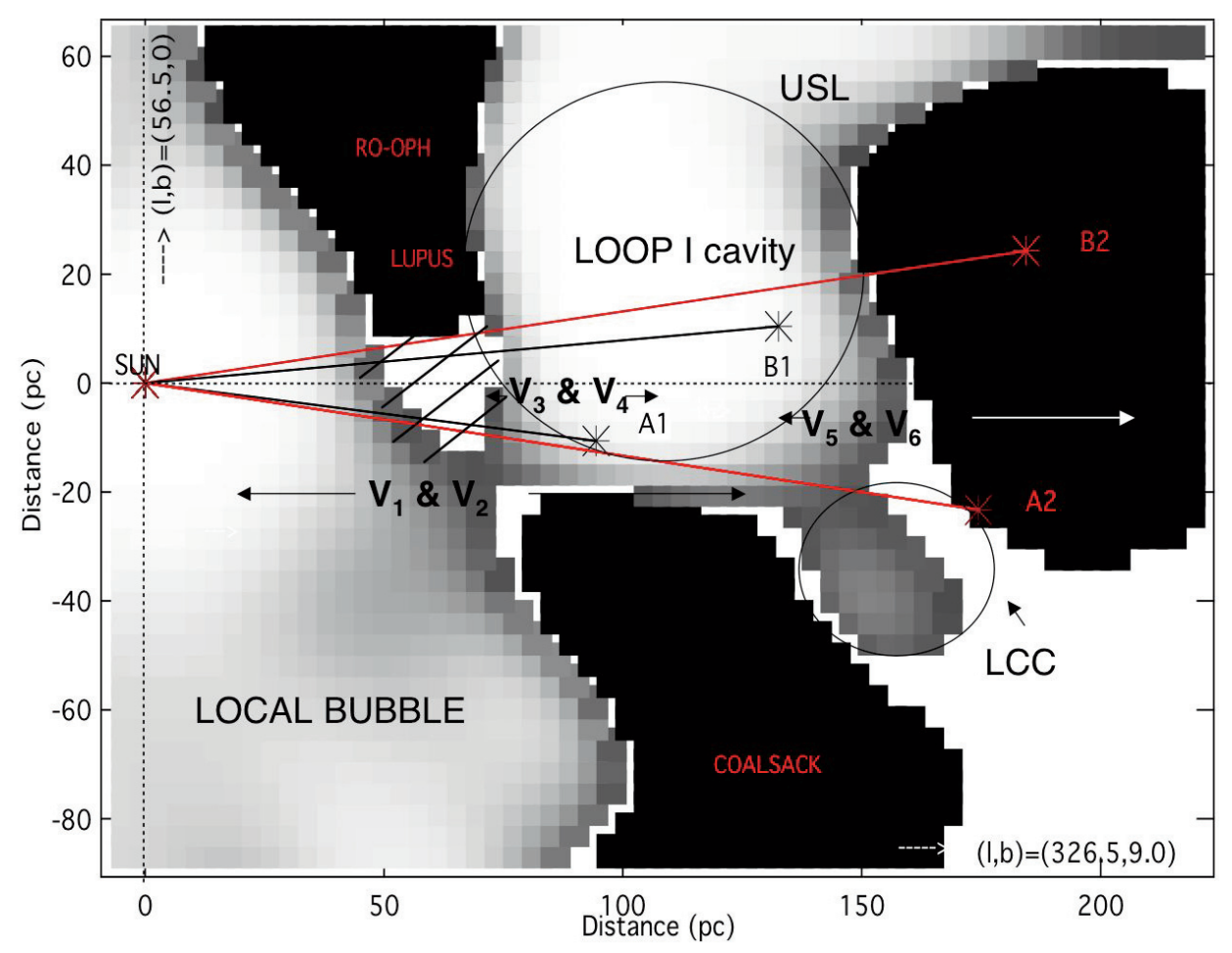

Fig. 6. Schematic map of the 2-D distribution of neutral (NaI) gas close to the galactic plane within 250 pc of the Sun. Dense gas is shown by dark shading, and hatch lines locate the area of interaction between the Local Bubble cavity and the adjacent Loop I superbubble. The approximate boundaries of the Upper Scorpius Loop (USL) and Lower Centaurus Loop (LCC) are also show. The distance estimates to the six cloud components $\left(\mathrm{V}_{1}\right.$ to $\left.\mathrm{V}_{6}\right)$ are indicated by arrows. The sight-lines to the target stars A1 (HD 128345), A2 (HD 127381), B1 (HD 138769) and B2 (HD 142256) are also shown.

same 4 UV absorption lines along the more distant "A2" sightline with column densities that are within a factor $\sim 2$ of those derived for the foreground "A1" sight-line.

If we assume that sulfur is undepleted with a solar abundance relative to hydrogen in the local ISM, then using $\log N(\mathrm{SII}) \sim 14.6 \mathrm{~cm}^{-2}$ for the $V \sim-15 \mathrm{~km} \mathrm{~s}^{-1}$ cloud towards "A1", we derive an equivalent hydrogen column density of $\log N(\mathrm{HI}+\mathrm{HII}) \sim 19.3 \mathrm{~cm}^{-2}$ for this absorption component. This value is very similar to that derived by Welsh et al. (1994) and Sfeir et al. (1999) for the neutral gas wall that defines the boundary to the local cavity in most galactic directions. Both Centurion \& Vladilo (1991) and Egger \& Aschenbach (1995) have also presented arguments for an increase in the gas column density for stars with (revised Hipparcos) distances $>80 \mathrm{pc}$ in the direction of Loop I, which indicates the presence of an interaction between our own local cavity and the adjacent Loop I superbubble. Therefore, we believe that the $V \sim-15 \mathrm{~km} \mathrm{~s}^{-1}$ cloud defines the boundary to the LB cavity in this direction, thus placing all of our 4 target stars just outside the inner confines of the local cavity. The placement of the $V \sim-15 \mathrm{~km} \mathrm{~s}^{-1}$ cloud at $\sim 90 \mathrm{pc}$ and its identification with that of the neutral boundary to the local cavity is verified by the 2-D density map shown in Fig. 6.

Although we have refered to the $V \sim-15 \mathrm{~km} \mathrm{~s}^{-1}$ component as a cloud boundary, there is ample evidence that this wall of gas is highly fragmented and in some sight-lines absorption is completely absent. For example, there are several narrow sight-lines with very low-density gas in this direction that have been named the Lupus interstellar tunnels (Welsh et al. 1994). They are revealed by the complete lack of detectable $\mathrm{NaI}$ and CaII absorption (to very low levels) along the directions towards the angularly-close stars of HD $142669(d=125 \mathrm{pc})$, $\operatorname{HD} 132058(d=161 \mathrm{pc})$ and $\operatorname{HD} 138690(d=176 \mathrm{pc})$ (Crawford 1991). We shall discuss the possible cause of this fragmentation of the local cavity boundary with respect to an interaction with the expansion of Loop I in the section dealing with the $V \sim-21 \mathrm{~km} \mathrm{~s}^{-1}$ absorption component.

\section{The $V \sim-10 \mathrm{~km} \mathrm{~s}^{-1}$ cloud}

A very weak neutral cloud with a velocity of $V \sim-9.7 \mathrm{~km} \mathrm{~s}^{-1}$ is revealed in the NaI absorption profile recorded towards "A1" and also in the NaI observations towards the more distant "A2". It is also not present in the UV line-profiles of $\mathrm{CI}$ or CII along either sight-line. However, gas with a similar velocity is detected in the UV profiles of FeII, SII and AlII towards both "A1" and "A2", but with column densities that differ by large factors between each sight-line. This may indicate that either appreciable gas density inhomogeneity is present over small angular scales in this cloud, or that the cloud extends for some distance beyond the "A1" sight-line in the direction towards "A2". We can estimate the minimum distance to this (neutral and partially ionized) cloud component to be $\sim 90 \mathrm{pc}$ (based on the non-detection of $\mathrm{NaI}$ over the 83 pc distance to HD 118991 by Lallement 2003), and thus it seems likely that this cloud may be loosely physically associated with the $V \sim-15 \mathrm{~km} \mathrm{~s}^{-1}$ gas discussed previously. These two clouds could exist in the form of sheets of gas moving with similar 
Table 3. Summary of cloud component physical characteristics.

\begin{tabular}{cccccl}
\hline \hline Cloud name & $\begin{array}{c}\text { Cloud velocity } \\
\left(\mathrm{km} \mathrm{s}^{-1}\right)\end{array}$ & $\begin{array}{c}\text { Cloud temperature } \\
(\mathrm{K})\end{array}$ & $\begin{array}{c}\log N(\mathrm{HI}+\mathrm{HII}) \\
\mathrm{cm}^{-2}\end{array}$ & $\begin{array}{c}\text { Cloud distance } \\
(\mathrm{pc})\end{array}$ & Ionization state \\
\hline $\mathrm{V}_{1}$ & -32 & $2800-22000$ & $18.3-18.5$ & $5-133$ & P.I. + H.I. \\
$\mathrm{V}_{2}$ & -21 & $2000-50000$ & $18.7-19.0$ & $5-95$ & P.I. + H.I. \\
$\mathrm{V}_{3}$ & -15 & $5500-15000$ & $18.8-19.3$ & $83-95$ & N + P.I. \\
$\mathrm{V}_{4}$ & -10 & $600-15000$ & $18.3-19.2$ & $83-95$ & N + P.I. \\
$\mathrm{V}_{5}$ & -1 & $1500-15500$ & $>19.2$ & $125-160$ & N + P.I. + H.I. \\
$\mathrm{V}_{6}$ & +3 & $850-6000$ & $>19.4$ & $125-170$ & P.I. \\
\hline
\end{tabular}

$\mathrm{N}=$ neutral, P.I. $=$ Partially Ionized, H.I. = Highly Ionized.

velocities, or represent gas that has been highly disturbed by the expansion of the Loop I superbubble into the local cavity. If we assume sulfur to be undepleted in the $V \sim-10 \mathrm{~km} \mathrm{~s}^{-1} \mathrm{ab}-$ sorption component, we derive an equivalent hydrogen column density of $\log N(\mathrm{HI}+\mathrm{HII}) \sim 19.2 \mathrm{~cm}^{-2}$, which is very similar to that derived for the $V \sim-15 \mathrm{~km} \mathrm{~s}^{-1}$ cloud.

\section{The $V \sim-1 \mathrm{~km} \mathrm{~s}^{-1}$ and $+3.0 \mathrm{~km} \mathrm{~s}^{-1}$ neutral clouds}

We have detected strong NaI, CI and CII neutral gas absorption at an average velocity of $V \sim-0.8 \mathrm{~km} \mathrm{~s}^{-1}$ solely along the more distant $176 \mathrm{pc}$ sight-line towards "A2". This absorption component is also observed at a similar velocity in many of the higher ionization UV absorption lines detected towards "A2", which sample warmer and (partially) ionized interstellar regions. In the cases of the SII and AlII lines an additional component at $V \sim+3.0 \mathrm{~km} \mathrm{~s}^{-1}$ is required to better fit their absorption profiles. The doppler $b$-values for the neutral lines of CI and CII detected at $V \sim-1 \mathrm{~km} \mathrm{~s}^{-1}$ towards "A2" imply respective temperature upper limits of $<6000 \mathrm{~K}$ for this gas component. These temperature estimates are consistent with that of $<6500 \mathrm{~K}$ derived from our fits of the high resolution NaI D-lines recorded towards "A2". Fits of the $V \sim$ $-1 \mathrm{~km} \mathrm{~s}^{-1}$ absorption component in the profiles of the SII, FeII and AlII lines indicate that saturation is present and thus both the best-fit doppler $b$-values and the associated component column densities are thus not well constrained. However, it is clear from these data that the $V \sim-1 \mathrm{~km} \mathrm{~s}^{-1}$ component has a high column density and consists of appreciable amounts of both cold neutral and warmer partially-ionized gas. The $V \sim+3.0 \mathrm{~km} \mathrm{~s}^{-1}$ component seems to possess no measurable cold and neutral gas, consisting mostly of warm and partially ionized gas.

A minimum distance of $\sim 125 \mathrm{pc}$ can be placed to the dense $V \sim-1 \mathrm{~km} \mathrm{~s}^{-1}$ cloud through the non-detection of NaI absorption at this velocity towards the angularly-close stars of HD 125238 ( $d=108$ pc, Welsh et al. 1994) and HD 130807 $(d=125 \mathrm{pc}$, Crawford 1991). However, dense gas with a velocity of $V \sim-1 \mathrm{~km} \mathrm{~s}^{-1}$ has been detected widely across this region in the absorption profiles of $\mathrm{NaI}$ and $\mathrm{CaII}$ towards many stars with distances $>160$ pc (Crawford 1991, 2000). Therefore we can conservatively place a distance estimate of $\sim 155 \mathrm{pc}$ to this cloud along sight-line "A". Based on the SII column density value for the $V \sim-1 \mathrm{~km} \mathrm{~s}^{-1}$ component, an equivalent hydrogen column density of $\log N(\mathrm{HI}+\mathrm{HII})>19.5 \mathrm{~cm}^{-2}$ can be derived. It seems highly likely that this cloud represents the far boundary to the Loop I superbubble cavity in this galactic direction, as shown in Fig. 6. Thus, the hot (X-ray emitting) rarefied gas of this superbubble is therefore seen to be contained between the $V \sim-15 \mathrm{~km} \mathrm{~s}^{-1}$ boundary to the local cavity at $\sim 90 \mathrm{pc}$ and the $V \sim-1 \mathrm{~km} \mathrm{~s}^{-1}$ gas cloud at $\sim 155 \mathrm{pc}$. Hence, our target star HD 127381 ("A2") at a distance of $176 \mathrm{pc}$ lies beyond both of the LB and Loop I interstellar cavities (as shown in Fig. 6).

Based on similar NaI detection arguments, it seems likely that the $V \sim+3.0 \mathrm{~km} \mathrm{~s}^{-1}$ is also located at similar distance to the $V \sim-1 \mathrm{~km} \mathrm{~s}^{-1}$ component. We note that Crawford (2000) has found that $\mathrm{NaI}$ gas clouds with positive velocities are only detected towards stars with distances $>170 \mathrm{pc}$ in this direction, which is interpreted as a net flow of gas away from the Sco-Cen association towards the galactic center. This would suggest that the $V \sim-1 \mathrm{~km} \mathrm{~s}^{-1}$ cloud at $\sim 155 \mathrm{pc}$ represents the transition region between gas with negative velocities that is flowing towards the local cavity, and gas with positive velocities that is receding way from the neutral boundary to Loop I and flowing into the next adjacent interstellar (Loop IV) cavity.

\section{The $V \sim-21 \mathrm{~km} \mathrm{~s}^{-1}$ cloud}

Gas absorption with an average velocity of $V \sim-20.9 \mathrm{~km} \mathrm{~s}^{-1}$ is seen in the UV line-profiles of FeII, SII and AlII along both " $A$ " sight-lines, thus placing its distance $<95 \mathrm{pc}$. Gas with a similar velocity has also been detected in the CaII observations of the angularly-close stars HD $133955(d=125 \mathrm{pc})$ by Crawford (1991) and HD $119921(d=131 \mathrm{pc})$ by Centurion \& Vladilo (1991), thus supporting the distance placement of $<100 \mathrm{pc}$ for this gas. This cloud seems to be deficient in neutral gas (i.e. it is not detected in the CI, ClI or NaI lines along either of the "A" sight-lines) and the doppler-widths required to fit the UV lines generally suggest a gas temperature $<12000 \mathrm{~K}$ for this component. If we assume that sulfur is undepleted in this absorption component towards "A1", then we derive an equivalent hydrogen column density of $\log N(\mathrm{HI}+\mathrm{HII}) \sim 19.0 \mathrm{~cm}^{-2}$ for this cloud. We note that the predicted projected velocity of the very local $(d<5 \mathrm{pc})$ interstellar "G-cloud" in the direction of the "A" sight-line is $\sim-21 \mathrm{~km} \mathrm{~s}^{-1}$ (Lallement \& Bertin 1992), which would apparently support the notion that we have detected absorption from very local gas long both sight-lines. However, we will presently delay a discussion on this point until Sect. 4.1.2. 


\subsubsection{The highly ionized gas towards HD 12738 (“A2")}

The major discovery along this sight-line is the detection of absorption from the high ions of CIV and NV (but not SiIV) towards HD 127381 ("A2"). This absorption is detected at a similar velocity $\left(V \sim-1.0 \mathrm{~km} \mathrm{~s}^{-1}\right)$ in both of the high ion lineprofiles, with an additional weaker component being detected at $V=-23.8 \mathrm{~km} \mathrm{~s}^{-1}$ solely in the CIV absorption profiles. We find that no contribution from highly ionized gas associated with the previously discovered absorption components covering the velocity range of -10 to $-18 \mathrm{~km} \mathrm{~s}^{-1}$ is required to fit these high ion profiles. Given the similarity between the presently observed high ion velocities and the $V \sim-1 \mathrm{~km} \mathrm{~s}^{-1}$ and $V \sim-21 \mathrm{~km} \mathrm{~s}^{-1}$ components discussed previously, it perhaps would seem likely that this high ion absorption is associated with the same interstellar gas clouds. However, how can such highly ionized (and high temperature) gas seemingly spatially co-exist with the far colder neutral interstellar gas at $V \sim-1 \mathrm{~km} \mathrm{~s}^{-1}$ that we have identified in the previous section?

Typically the profile widths of CIV, SiIV and NV absorption lines have been found to be quite broad in the general interstellar medium $\left(b>10 \mathrm{~km} \mathrm{~s}^{-1}\right.$ ), indicating their origin in high temperature, often collisionally ionized regions (Savage et al. 2001). However, we have found surprisingly small values $\left(<4.5 \mathrm{~km} \mathrm{~s}^{-1}\right)$ for the doppler $b$-values for the CIV and $\mathrm{NV}$ absorption component seen at $V \sim-1.0 \mathrm{~km} \mathrm{~s}^{-1}$ towards HD 127381. In particlar, the $b$-value required to fit the CIV absorption profiles imply an upper limit of only $\sim 2500 \mathrm{~K}$ for the (thermal) temperature of this gas. We note that it may not be coincidental that with the advent of recent access to high resolution spectrographs in the space ultraviolet several other authors have also reported detecting similarly narrow components in the interstellar profiles of the CIV and SiIV lines observed towards several more distant targets (Fox et al. 2003; Knauth et al. 2003). At such a low gas temperature these high ions cannot be produced via normal equlibrium collisional processes, and thus photo-ionization has been fowarded as the most likely explanation for their formation in the interstellar medium.

Local high ions are generally expected to form at the conductive interfaces between evaporating hot and cooler interstellar gas (Slavin 1989). This is a scenario that would seem to fit the physical situation in which CIV and NV gas is present at the same velocity as that of the neutral gas boundary to the Loop I cavity (i.e. $V \sim-1 \mathrm{~km} \mathrm{~s}^{-1}$ ), which interfaces with the hot X-ray emitting gas that resides within the inner regions of this superbubble cavity. However, the observed narrow line-widths of the presently observed high ion absorption profiles would seem to preclude any production mechanism that involves collisions and/or turbulent mixing at a putative conductive interface. Thus, our observations are in strong disagreement with many current theoretical models of evaporating conductive interfaces which predict far higher amounts of high ion absorption that should be observed at the four cloud (conductive) interfaces encountered over the 176 pc distance to "A2". Thus, we conjecture that either the present theoretical models are incorrect, or we have a very different physical situation present along sightline "A2".
We note that there is ample empirical evidence demonstrating that the Loop I superbubble contains hot $\left(T \sim 10^{6.5} \mathrm{~K}\right)$ and highly ionized X-ray emitting gas (Willingdale et al. 2003; Breitschwerdt et al. 2000). It has also been found that this hot gas is the probable source of the CIV, SiIV and NV absorption lines detected in the UV spectra of more distant background sources whose interstellar sight-lines pass through this superbubble (Sembach et al. 1997). Loop I also contains the many young OB stars of the Sco-Cen association that possess a large stellar wind-power and can potentially provide an appreciable source of (photo-)ionizing radiation for the surrounding interstellar gas. These early-type stars have, on average, distances $\sim 150 \mathrm{pc}$ (de Geus 1989), thus positioning many of them within the dense neutral gas Loop I boundary (as defined by the $V \sim-1 \mathrm{~km} \mathrm{~s}^{-1}$ cloud component). Based on these factors we believe that ionization contributions from both the hot X-ray emitting gas of the Loop I bubble and the stellar UV photons from the many early-type stars of the Sco-Cen OB association seem the most likely source of photo-ionization of the neutral gas at the Loop I boundary wall. However, we note that the photon flux from the winds of hot stars is greatly reduced for energies $>54 \mathrm{eV}$ and would thus not be able to explain the present detection of the NV line $(77.5 \mathrm{eV})$ towards HD 127381. Therefore, the ionization contribution from the X-ray emitting hot gas of Loop I must dominate the total local level of photoionization. The X-ray emission from the interior of this superbubble has been attributed to the interaction between shock waves generated by the most recent supernova event within the Sco-Cen association and the ambient neutral gas which heats up to a temperature of $\sim 3 \times 10^{6} \mathrm{~K}$ (Egger \& Aschenbach 1995). It also seems highly probable that the presently observed high ions are formed on the inner side of the boundary wall of neutral gas at $\sim 150 \mathrm{pc}$ that faces towards the inner regions of the hot Loop I cavity.

Theoretical models that invoke photo-ionization due to a classic HII region (Howk \& Savage 1999) or a stellar wind-driven bubble (Weaver et al. 1977) can be presently discounted since they generally predict columnn density ratios that are incompatible with those we have determined for $N(\mathrm{CIV}) / N(\mathrm{SiIV})$ and $N(\mathrm{CIV}) / N(\mathrm{NV})$. Instead, we favor a model similar to that described by Slavin \& Frisch (2002) in which an interstellar cloud is photo-ionized by both nearby OB stellar photons and the diffuse X-ray emission from an ambient hot (several million degree $\mathrm{K}$ ) gas. Although the Slavin and Frisch calculations were specifically aimed at reproducing the physical conditions in the very local interstellar cloud, they can quantitatively match our presently observed column density ratios of both $N(\mathrm{CIV}) / N(\mathrm{NV})=7.3$ and $N(\mathrm{CIV}) / N(\mathrm{SiIV})>22$ for the $V \sim-1 \mathrm{~km} \mathrm{~s}^{-1}$ cloud component. Interestingly, this model also predicts very small amounts of absorption due to the interstellar SiIII ( $\lambda 1206 \AA$ ) line, which is in agreement with our detection limit of $<2 \mathrm{~m} \AA$ for its equivalent width along sight-line "A2". We suggest that earlier claims for the detection of appreciable local absorption from the SiIII line (Holberg et al. 1999; Gry \& Jenkins 2001) may be due to the measurement of photospheric absorption lines which are often difficult to identify in the spectra of hot white dwarf and very early B-type stars. 
Although we favor a photo-ionization model for the presently observed CIV and NV lines at $V \sim-1 \mathrm{~km} \mathrm{~s}^{-1}$, we cannot rule out other types of production models that require special cases of non-equilibrium conditions (Breitschwerdt \& Schmutzler 1994), or the effects of magnetic field containment (Edgar \& Chevalier 1986; Borkowski et al. 1990). In particular, non-equilibrium time-dependent shock-heated cooling models allow the formation of interstellar CIV at very low temperatures. For example, the model of Breitschwerdt (2001) predicts very cold, high ionization lines for the case of a SNR that has expanded into a low density interstellar cavity, a situation that certainly resembles that of the Loop I superbubble expanding into the adjacent rarefied LB cavity. In this case, delayed recombination is a direct consequence of fast adiabatic expansion in which the high ion states are in a state of extreme non-equilibrium and the lines of CIV and NV can be essentially "frozen" into the plasma.

We have also detected a weak CIV absorption component at $V=-23.9 \mathrm{~km} \mathrm{~s}^{-1}$ along the sight-line towards "A2", which is close to the velocity of the $V \sim-21 \mathrm{~km} \mathrm{~s}^{-1}$ gas cloud discussed previously. The small value of column density of $\log N(\mathrm{CIV})=12.3 \mathrm{~cm}^{-2}$ measured for this component towards "A2" would preclude our detection of a similar absorption component that may be present in the other high ionization lines of NV or SiIV. The $V \sim-23.9 \mathrm{~km} \mathrm{~s}^{-1}$ absorption component also has a small doppler-width, indicative of a thermal gas temperature of $<5500 \mathrm{~K}$, which is of a very similar value to that observed in the main $V \sim-1 \mathrm{~km} \mathrm{~s}^{-1}$ component of the CIV line. This similarity in doppler-width would perhaps favor a common ionization mechanism for both of the CIV components (i.e. photo-ionization or non-equilibrium processes). However, knowledge of the distance to the $V \sim-23.9 \mathrm{~km} \mathrm{~s}^{-1}$ gas would help in deciding which ionization mechanism is prefered, and thus we now present such a discussion.

\subsubsection{The distance to the $V \sim-21 \mathrm{~km} \mathrm{~s}^{-1}$ cloud}

The detection of gas with a velocity of $V \sim-21 \mathrm{~km} \mathrm{~s}^{-1}$ in the UV lines along both "A" sight-lines and in the CaII lines towards several angularly-close stars, implies a distance $<95 \mathrm{pc}$ to this cloud. Its observed velocity is very close to the projected velocity value for the very local $(d<5$ pc) interstellar "G-cloud" in this direction of $\sim-21 \mathrm{~km} \mathrm{~s}^{-1}$ (Lallement \& Bertin 1992). Also, inspection of Table 2 reveals that an absorption component with an average velocity of $V \sim-25.4 \mathrm{~km} \mathrm{~s}^{-1}$ has been detected in the UV lines along both "B" sight-lines, which is very similar to the projected velocity of the "G-cloud" in this direction of $V \sim-24.5 \mathrm{~km} \mathrm{~s}^{-1}$. This similarity between the observed component and projected "G-cloud" velocities for both "A" and "B" sight-lines, and its detection at distances $<95 \mathrm{pc}$, argues strongly for an association with very local gas. However, there are several other factors that argue against such a distance placement. Firstly, the derived value of $\log N(\mathrm{HI}+\mathrm{HII}) \sim 19.0 \mathrm{~cm}^{-2}$ for the absorption component observed along sight-line " $\mathrm{A}$ " is a factor 3-4 higher than that normally found for very local clouds (Lallement et al. 1995), although we note that the hydrogen column density of the "G-cloud" has yet to be determined. In addition, the value of $\log N(\mathrm{FeII})>13.1 \mathrm{~cm}^{-2}$ for this component along both " $A$ " and " $B$ " sight-lines is several times greater than that measured for stars with distances $<10 \mathrm{pc}$ in this general direction (Redfield \& Linsky 2002). Our presently measured high values of $N(\mathrm{FeII})$ for this cloud component are much closer to those determined by Lehner et al. (2003) for stars in the $50-150 \mathrm{pc}$ distance range. Although the high values of $N(\mathrm{FeII})$ could be due to density gradients that exist in the "G-cloud" (perhaps caused by the effects of local heating/ionization or element overabundance), or by the "G-cloud" physically extending for large distances towards the direction of the Galactic center, we note that recent observations have raised concerns over the actual existence of the "G-cloud" itself.

Crawford (2001) has presented ultra-high resolution CaII absorption data towards 8 stars with distances $<200 \mathrm{pc}$ that have failed to provide convincing evidence for the detection of both the local interstellar cloud (LIC) and the "G-cloud". The relatively weak LIC absorption was masked by absorption components arising in more distant clouds that possessed local ISM-like velocities. The non-detection of the "G-cloud" (if infact it really does exist as a separate entity to the LIC) in the CaII observations is explained by its spatial extent not extending to galactic latitudes $>+15^{\circ}$ in this general direction of the sky. Furthermore, in the model of the interaction between the Local Bubble and the expanding Loop I superbubble of Breitschwerdt et al. (2000), a general flow of small and compact cloudlets that have been expelled from this region by Rayleigh-Taylor instabilities are assumed to explain the presence of neutral and partially ionized "local fluff" cloudlets that should exist throughout the whole of the fully ionized Local Bubble region. Therefore, coincidence between the absorption velocities of cloud components with quite different distances may be commonplace in the local ISM. If the measured velocity of the $V \sim-21 \mathrm{~km} \mathrm{~s}^{-1}$ component is just mere coincidence with that of the very local "G-cloud", then its distance could be in the 50-95 pc range. This would then locate such gas near to the interaction region between the local cavity and Loop I and could account for the apparently anomalous values of $N(\mathrm{HI}+\mathrm{HII})$ and $N(\mathrm{FeII})$ we have derived for this component when compared to similar measurements of the very local gas.

We also note from Table 2 that high ion absorption has only been detected along the "A2" and "B2" sight-lines. In the case of $\mathrm{CIV}$, there is a small velocity difference of $-2.9 \mathrm{~km} \mathrm{~s}^{-1}$ between the observed high ion component velocity of $-23.8 \mathrm{~km} \mathrm{~s}^{-1}$ and that of the $V \sim-20.9 \mathrm{~km} \mathrm{~s}^{-1}$ component seen in the lower ionization UV lines towards "A2". In the case of "B2", the observed velocity of the CIV component is identical to that measured for "A2", whereas the $V \sim-20.9 \mathrm{~km} \mathrm{~s}^{-1}$ component seen in the other UV lines is shifted to $V \sim-25.4 \mathrm{~km} \mathrm{~s}^{-1}$. Although this high ion absorption is weak in both sight-lines, this small velocity difference between the high and lower ionization ions may be real and indicate that these clouds are different physical entities. If the formation of these ions were associated with the very local "G-cloud" then we would have expected to detect their presence in the spectra of both of the foregound sight-lines towards "A1" and "B1". However, we have failed to detect such high 
ion absorption components in these foreground sight-lines, thus placing their origin at a distance $>133 \mathrm{pc}$ (i.e. the distance to "B1"). Therefore, this would place the $V \sim-23.9 \mathrm{~km} \mathrm{~s}^{-1}$ cloud close to a known potential source of photo-ionization (i.e. Loop I and the Sco-Cen OB stars), and provide an explanation for the observed narrow doppler-width of this high ion velocity component. However, other production mechanisms that involve the "freezing" of high stages of ionization in a very low density gas that adiabatically cools before recombining clearly also cannot be ruled out at present (Breitschwerdt \& Schmutzler 1994).

In summary, we favor the placement of the $V \sim-21 \mathrm{~km} \mathrm{~s}^{-1}$ component observed in the lower ionization UV lines at a distance $<10 \mathrm{pc}$, but note that subsequent higher spectral resolution observations may reveal that this component consists of more distant but similar velocity gas that is blended in our present data. We also favor the $V \sim-23.9 \mathrm{~km} \mathrm{~s}^{-1}$ high ionization cloud as a separate entity to the lower ionization $V \sim-21 \mathrm{~km} \mathrm{~s}^{-1}$ gas, with a distance in the 135-150 pc range close to a source of strong photo-ionization.

\subsection{The sight-line towards $H D 138769$ and $H D$ 142256: The neutral and low-ionization gas along sight-line " $B$ "}

\section{The $V \sim-2 \mathrm{~km} \mathrm{~s}^{-1}$ and $V \sim+3 \mathrm{~km} \mathrm{~s}^{-1}$ clouds}

We have failed to detect cold and dense neutral gas absorption at any velocity in all of the NaI, CI and ClI absorption lines observed along the $133 \mathrm{pc}$ interstellar sight-line towards the star HD 138769 ("B1"). However, strong absorption is detected in each of these neutral lines at $V \sim-2 \mathrm{~km} \mathrm{~s}^{-1}$ along the $186 \mathrm{pc}$ sight-line to the more distant "B2". Inspection of the doppler line-widths required to fit both the $\mathrm{NaI}$ and $\mathrm{CI}$ lines indicate a gas temperature of $<4000 \mathrm{~K}$ for this component. We note that no $\mathrm{NaI}$ has been detected at this velocity towards the angularly-close stars of HD $140602(d=173 \mathrm{pc})$ and HD $138690(d=174 \mathrm{pc})$ by Crawford (2000). This places a distance limit of $\sim 180 \mathrm{pc}$ to the cold and dense gas of the $V \sim-2 \mathrm{~km} \mathrm{~s}^{-1}$ cloud in this galactic direction. We note that this is most probably the same gas cloud (identified previously at $V \sim-1 \mathrm{~km} \mathrm{~s}^{-1}$ ) detected along the "A2" sight-line with a distance of $\sim 155 \mathrm{pc}$ that defines the outer boundary to the Loop I superbubble cavity. The slightly greater distance to this boundary along the "B" sight-line merely illustrates the contorted complexity of gas clouds in this galactic region.

However, we have also detected a weak absorbing cloud with a similar velocity to the $V \sim-2 \mathrm{~km} \mathrm{~s}^{-1}$ cloud component in the FeII, SII and AlII lines towards the foreground star "B1" ( $d=133 \mathrm{pc})$, which would seem to invalidate our previous distance placement of $\sim 180 \mathrm{pc}$ for gas with this low velocity. We note, however, that the derived column densities of the FeII, SII and AlII components of the $V \sim-2 \mathrm{~km} \mathrm{~s}^{-1}$ gas are significantly different between the "B1" and "B2" sightlines. Under the assumption that sulfur is undepleted in the ISM, we derive an equivalent total hydrogen column density of $\log N(\mathrm{HI}+\mathrm{HII})=19.2 \mathrm{~cm}^{-2}$ for the foreground $V \sim-2 \mathrm{~km} \mathrm{~s}^{-1}$ gas detected towards "B1", which is to be compared with a value at least 5 times greater for the same velocity cloud detected towards the more distant "B2". Similar conclusions can be gained from an inspection of the respective column densities of the other UV ions detected along both sight-lines. Inspection of Fig. 6 clearly shows that "B1" is located well within the confines of the low-density Loop I cavity and $\sim 30$ pc foreground to the cold dense cloud that defines the outer boundary to the supoerbubble, whereas "B2" lies well within the inner regions of this dense cloud. Given the size of the errors of gas cloud (and stellar target) distances discussed previously, we suggest that the gas at $V \sim-2 \mathrm{~km} \mathrm{~s}^{-1}$ detected foreground to HD 138769 ("B1") probably represents a warm and partially ionized cloud that is part of the fragmentation region at $\sim 90 \mathrm{pc}$.

An additional component with an average velocity of $V \sim+3 \mathrm{~km} \mathrm{~s}^{-1}$ is required to fit the SII lines detected towards "B2". This is most probably the same cloud identified at the same velocity discussed previously for the "A2" sight-line with a distance of $\sim 155 \mathrm{pc}$. Once again, it is only seen in the warmer and partially ionized gas revealed by the UV absorption lines and contains no detectable cold and neutral component.

\section{The $V \sim-11,-18,-25$ and $-32 \mathrm{~km} \mathrm{~s}^{-1}$ components}

We have detected warm and partially ionized gas in the UV lines of FeII, SII and AlII in 4 cloud components with average velocities of $-10.7,-17.8,-25.4$ and $-32.5 \mathrm{~km} \mathrm{~s}^{-1}$ along the "B1" sight-line. All but the $V \sim-17.8 \mathrm{~km} \mathrm{~s}^{-1}$ component are detected with similar velocities along the "B2" sight-line. We can immediately associate the gas at $V \sim-11 \mathrm{~km} \mathrm{~s}^{-1}$ with the $V \sim-10 \mathrm{~km} \mathrm{~s}^{-1}$ cloud of distance $\sim 90 \mathrm{pc}$ detected towards both "A" sight-lines which has been discussed in the previous section.

The $V \sim-18 \mathrm{~km} \mathrm{~s}^{-1}$ cloud is only detected towards the foreground "B1" sight-line. Taking into account sight-line velocity projection effects it is reasonable to assume that this is the same cloud detected at $V \sim-15 \mathrm{~km} \mathrm{~s}^{-1}$ along sightline " $A$ ". This is the cloud that we have previously argued defines the (fragmented) neutral boundary to the local cavity in this galactic direction. We note that, based on the value of sulfur column density derived towards "B1", the equivalent hydrogen column density value of $\log N(\mathrm{HI}+\mathrm{HII})=18.8 \mathrm{~cm}^{-2}$ is $40 \%$ lower than that derived for the corresponding sightline "A" velocity component. This reduced value of gas column density provides a clue as to why gas with a velocity of $\sim-18 \mathrm{~km} \mathrm{~s}^{-1}$ has not been detected along the more distant "B2" sight-line. As outlined in the discussion of the $V \sim-15 \mathrm{~km} \mathrm{~s}^{-1}$ component observed towards sight-line "A", we showed observational evidence for gaps and tunnels through the boundary cloud to the local cavity. It therefore seems likely that the sightline towards "B2" passes through such a "hole" in the neutral wall of the local cavity, thus explaining the lack of detectability of the $V \sim-15 \mathrm{~km} \mathrm{~s}^{-1}$ component in the sight-line towards "B2" (we aslo note that from a statistical standpoint our best-fits to the UV lines towards "B2" do not require a cloud at $V \sim-18 \mathrm{~km} \mathrm{~s}^{-1}$, although some small amount of absorption may well be present at this velocity). The smaller value of hydrogen column density derived towards " $\mathrm{B} 1$ " is also explained 
by that sight-line passing close to the this gap in the neutral gas wall of the local cavity at a distance of $\sim 90 \mathrm{pc}$.

Warm and partially ionized gas with an average velocity of $V \sim-25.4 \mathrm{~km} \mathrm{~s}^{-1}$ has been detected along both "B" sightlines in the UV line-profiles of FeII, SII and AlII. We note that the projected velocity of the purported "G-cloud" along these sight-lines is $\sim-24.5 \mathrm{~km} \mathrm{~s}^{-1}$ (Lallement \& Bertin 1992). Based on the SII column density value of this absorption component derived from both the "B" sight-lines, we compute an equivalent hydrogen column density of $\log N(\mathrm{HI}+\mathrm{HII}) \sim 18.7 \mathrm{~cm}^{-2}$ for this gas cloud. This value is consistent with that derived previously from sight-line " $A$ " observations of gas absorption with a component velocity of $V \sim-21 \mathrm{~km} \mathrm{~s}^{-1}$. We also note the apparently high values of $N($ FeII $)$ measured along both "B" sightlines for this velocity component. Redfield \& Linsky (2002) and Lehner et al. (2003) have shown that the relative depletion of Fe in the local ISM is highly variable, differing by an order of magnitude between some local sight-lines. It would therefore appear that the relative depletion of Fe may be low towards Loop I, which could be explained by elemental enrichment due to the sputtering of dust grains caused by the joint action of supernova and stellar wind-driven shocks.

We have also detected a new absorption component in the UV line-profiles of FeII, SII and AlII along both "B" sight-lines with an average velocity of $V \sim-32.4 \mathrm{~km} \mathrm{~s}^{-1}$, thus placing its distance $<133 \mathrm{pc}$. No neutral gas with this velocity has been detected along either sight-line, thereby indicating that the cloud is located in more ionized regions. Based on the SII column density measured towards "B1" we derive an equivalent hydrogen column density of $\log N(\mathrm{HI}+\mathrm{HII}) \sim 18.5 \mathrm{~cm}^{-2}$ for this gas cloud. This column density is typical for diffuse and warm, partially ionized clouds that reside throughout the local cavity. Gas with a very similar velocity to the $V \sim-32.4 \mathrm{~km} \mathrm{~s}^{-1}$ cloud has also been observed through ultra-high resolution CaII observations of the sight-lines towards both $\alpha$ Oph $(d=14 \mathrm{pc})$ and $\gamma$ Oph $(d=29 \mathrm{pc})$ by Crawford \& Dunkin (1995) and Crawford et al. (1997), which lie $\sim 30^{\circ}$ away from the "B" sight-line direction. However, no absorption at this velocity has been detected towards any of the 4 stars of the $\rho$ Oph system $(d=$ $150 \mathrm{pc}$ ) that is situated much closer to the " $\mathrm{B}$ " sight-lines (Pan et al. 2004). Therefore, until further high-resolution absorption studies of many more sight-lines towards Loop I become available, we are currently unable to determine whether the $V \sim-32.4 \mathrm{~km} \mathrm{~s}^{-1}$ cloud is of very local origin $(d<14 \mathrm{pc})$ or whether it is situated much further away and thus closer to the Loop I cavity.

\subsubsection{The highly ionized gas towards HD 142256}

Our main finding along the "B2" sight-line is the detection of two absorption components with average velocities of $V \sim$ -22.8 and $-32.4 \mathrm{~km} \mathrm{~s}^{-1}$ in the high ionization line-profiles of the CIV and SiIV high ions. No associated NV absorption has been detected at these velocities, and no high ion gas absorption is detected in any of the profiles with velocities $>-15 \mathrm{~km} \mathrm{~s}^{-1}$. The doppler $b$-values required to fit these high ion absorption components indicate gas temperature upper limits (from both the SiIV and CIV profiles) of $50000 \mathrm{~K}$ for the $V \sim-22.8 \mathrm{~km} \mathrm{~s}^{-1}$ component and $20000 \mathrm{~K}$ for the $V \sim-32.4 \mathrm{~km} \mathrm{~s}^{-1}$ cloud. Although these $b$-values are larger than those found for the high ion absorption components detected along sight-line "A2", they are still significantly smaller than the doppler line-widths expected from production mechanisms that involve conductive interfaces or collisions.

We have found that the column density ratios of $N(\mathrm{CIV}) / N(\mathrm{SiIV})$ for both absorption components are similar (8.1 for the $V \sim-23 \mathrm{~km} \mathrm{~s}^{-1}$ component and 5.4 for the $V \sim-32.5 \mathrm{~km} \mathrm{~s}^{-1}$ component). This would suggest a common source of ionization. We also note that these ratios are about a factor of two greater than that of $3.6 \pm 1.3$ which has been reported for these high ions in the general ISM (Sembach $\&$ Savage 1992). These ratios are also at least a factor 4 less than that found for the main (photo-ionized) component at $V \sim-1 \mathrm{~km} \mathrm{~s}^{-1}$ observed towards HD 127381 (“A2"). As discussed earlier, high ions produced at an evaporating conductive interface between hot and cooler interstellar gas should have doppler line-widths indicative of a thermal gas temperature of $\sim 100000 \mathrm{~K}$ (i.e. $b>10 \mathrm{~km} \mathrm{~s}^{-1}$ ), which is clearly not the case for either of the presently detected high ion components. The CIV column density values for both components are, however, similar to those predicted by several different theoretical models of high ion production at a single conductive interface with properties similar to diffuse gas clouds found within the very local ISM (Slavin 1989; Bohringer \& Hartquist 1987). Furthermore, we also note that all of these conduction-front models predict levels of SiIV absorption that are more than a factor 10 lower than the presently detected column density towards HD 142256.

Clearly, precise knowledge of the distance to these two, high ion bearing, clouds would be helpful in determining possible ionization production mechanisms. We have already dealt with a possible distance determination to the $V \sim-23.9 \mathrm{~km} \mathrm{~s}^{-1}$ absorption component detected in the CIV profile in Sect. 4.1.2 and we are only able to place an upper limit of $d<133 \mathrm{pc}$ for the $V \sim-32.5 \mathrm{~km} \mathrm{~s}^{-1}$ high ion component. Again, we note that there are small velocity differences between the component velocities observed for the low ionization UV lines and the high ion components revealed in the CIV and SiIV lines. It may be possible, as argued for the $V \sim-1 \mathrm{~km} \mathrm{~s}^{-1}$ cloud observed towards "B1", that we are actually observing completely separate clouds with different distances but similar velocities. Clearly, the placement of the highly ionized gas clouds in the $135-150 \mathrm{pc}$ distance range would situate them close to a strong source of photo-ionization that could then explain their observed narrow doppler line-widths. Finally, we note that we have not detected the very strong high ionization component observed at $V \sim-1 \mathrm{~km} \mathrm{~s}^{-1}$ in the $\mathrm{CIV}$ and $\mathrm{NV}$ lines along sight-line "A2". This could be due to "B2" being shielded from the source of ionization that is responsible for the "A2" high ions. Unfortunately, until higher spectral resolution observations are recorded towards many more sight-lines in this galactic direction we are are unable to comment further on this conjecture.

In Table 3 we summarize the approximate physical characteristics of the six absorption components detected along 
sight-lines "A" and "B". This table lists the average velocity of each cloud component, the minimum and maximum temperature of each cloud component derived from the doppler $b$-values for line-species listed in Table 2, the range of hydrogen column density, $N(\mathrm{HI}+\mathrm{HII})$ derived from values of $N(\mathrm{SII})$ listed in Table 2 for each cloud component, the minimum and maximum distance to each cloud and the ionization state of the cloud component defined for neutral $(\mathrm{N})$, partially ionized (P.I.) and highly ionized (H.I.) gas. These results are also depicted schematically in Fig. 6 such that the distance estimates of the clouds given in Table 3 can be compared with the neutral boundary contours of the local cavity and the Loop I superbubble. We note that the temperature estimates for several of these clouds are not well constrained due to the existence of both neutral and ionized species with similar absorption component velocities. In reality, the ionized gas most likely resides on the outer skin of an inner neutral interstellar cloud.

\subsubsection{Summary conjectures}

Our main finding in this Paper is the detection of highly ionized absorption lines in sight-lines towards the Loop I superbubble that are characterized by narrow doppler line-widths, suggesting that their formation is caused either by non-equilibrium processes or by a strong nearby source of photo-ionization. These observations are incompatible with theoretical models that predict the formation of highly ionized species at evaporating conductive interfaces. Unfortunately, our present absorption data are unable to distinguish between any of the theoretical models that we have invoked to explain the formation of these highly ionized species. Firstly we remind readers that our present observations of highly ionized gas along only two sight-lines may not be typical of the rest of the local cavity. However, with this caveat in mind, we now explore other observations of the local gas that can aid us in both making an informed choice between possible high ion line production models and which may provide some insight into our speculation on the physical state of the gas within the Local Bubble cavity.

The original idea of a local plasma in a high state of non-equilibrium was presented by Breitschwerdt \& Schmutzler (1994) in order to explain the the non-detection of line emission in the diffuse EUV background signal by Jelinsky et al. (1995). These emission lines, whose absence has recently been confirmed to even lower limits by the NASA CHIPS mission (Hurwitz et al. 2005), should be present if local hot gas (with a temperature of $\sim 10^{6} \mathrm{~K}$ as derived from observations of the soft X-ray background) is in collisionally ionized equilibrium. Other authors have also suggested that the very local gas may be out of equilibrium based on the observed anomalous fraction of helium ionization in the local clouds (Lyu \& Bruhweiler 1996). On the other hand, this class of non-equlibrium model is incompatible with the levels of OVI ( $\lambda 1032 \AA)$ and CIII ( $\lambda 977 \AA)$ emission lines observed in the diffuse far UV emission spectra of the local gas (Shelton 2003; Welsh et al. 2002). However, we note that observations of this diffuse far UV emission are very few in number and each observation covers only a very small area of the sky. Thus, if emission from the highly ionized local gas is restricted to small spatial regions (i.e. over a small fraction of an interstellar cloud's surface area) then the previous arguments can be discounted. This scenario of limited regions of high ion absorption (caused by conduction being quenched by a magnetic field, Cox \& Helenius 2003), has been suggested by Oegerle et al. (2005) to explain the $50 \%$ detection rate of OVI absorption in a FUSE survey of 25 sight-lines through the local ISM. However, we note (significantly) that the dopplerwidths of the OVI absorption lines observed in this survey are consistent with that expected for a gas with a thermal temperature of $\sim 3.5 \times 10^{5} \mathrm{~K}$. This, of course, is at variance with the small doppler-widths we have found for the CIV, NV and SiIV absorption lines. Alternately, the interpretation of the diffuse soft X-ray background spectra provides a different picture. The ROSAT maps of the the $0.25 \mathrm{keV}$ diffuse X-ray emission of Snowden et al. (1998) show little evidence to support the notion of a patchy spatial distribution for local hot gas emission. However, there is a growing awareness that these maps may contain a non-negligible emission contribution from the very local interplanetary medium, such that a re-assessment of previously held shibboleths concerning the soft X-ray background may be needed (Lallement 2004). This view is supported by the recent CHANDRA spectral observations by Smith et al. (2005) that suggest that measurement of any soft X-ray signal generated solely in the local cavity is extremely difficulty to distinguish from that of heliospheric generated X-rays. It appears that the OVII/OVIII line emission ratio may be the key to distinguishing between extremely local and any more distant hot gas emission. In an attempt to reconcile many aspects of these seemingly contradictory data, Shelton (2003) has suggested that the OVI emitting plasma and the X-ray emissive plasma may reside in distinct and separate regions of the Local Bubble and are not mixed in a single plasma, whether such gas is in equilibrium at $T \sim 10^{6} \mathrm{~K}$ or if it is highly over-ionized.

Photo-ionization of the local interstellar gas by nearby early-type stars (i.e. $\epsilon$ and $\beta \mathrm{CMa}$ ) and hot white dwarfs is known to be the most important factor in determining the intensity of the local EUV radiation field (Vallerga \& Welsh 1995). In a FUSE absorption survey of 30 stars within 200 pc by Lehner et al. (2003), it was determined that photo-ionization is a major influence on the physical condition of the local gas, and that photo-ionization conditions may not be constant throughout the Local Bubble. This may explain our present observations that have sight-lines that point in the direction of an appreciable source of photo-ionization (i.e. the ScoCen/Loop I complex). Sophisticated photo-ionization models, such as that of Slavin \& Frisch (2002), have attempted to describe the expected ionization state of the local interstellar cloud using a combination of radiation sources that include nearby hot stars, the diffuse emission from the soft X-ray background and emission from a possible evaporative boundary between the local cloud and the local hot gas. Although some success has been made in describing the expected column density ratios for local high ion absorption in this cloud for the sight-line towards $\epsilon \mathrm{CMa}$, there have been no other detections of high ion absorption in any sight-line within $100 \mathrm{pc}$ to test this 
model further. Although our present results generally support the predictions of this model, we have argued that the presently detected high ion absorption is not associated with the local cloud and perhaps originates in clouds closer to the Loop I superbubble. In addition, the model does not comment on the expected line-widths of local-cloud generated high ion absorption lines. Clearly until many more sight-lines throughout the Local Bubble region have been sampled using high resolution UV absorption spectra, we are presently unable to comment further on the physical condition of the local highly ionized gas. This is particularly relevant to the recent failure of the HST-STIS instrument, in that no future high resolution UV spectrograph is presently planned for launch by NASA.

\section{Conclusion}

We have observed the interstellar sight-lines towards four nearby $(d<200 \mathrm{pc})$ early-type stars, lying in the direction towards the Loop I superbubble, with the E140M echelle grating of the HST-STIS instrument to obtain medium resolution $\left(R \sim 6.5 \mathrm{~km} \mathrm{~s}^{-1}\right)$ ultraviolet absorption-line spectra over the wavelength range 1150-1690 ̊. These data have been supplemented with high resolution $\left(R \sim 3 \mathrm{~km} \mathrm{~s}^{-1}\right)$ visible absorption spectra of the NaI D-lines at $5890 \AA$.

We have presented an analysis of 10 of the many interstellar absorption lines detected along these sight-lines which are deemed to be representative of the neutral and ionized gas that may be present in the local ISM. The major finding from these observations is the detection of high ionization absorption, associated with the lines of CIV, NV and SiIV, whose best-fits require surprisingly small doppler-width values that infer very low temperatures for this highly ionized gas. This is in direct contradiction with many theoretical models that predict appreciable amounts of high ions formed in the evaporating gas at the conductive interfaces of interstellar clouds. Our presently sampled sight-lines cross at least four of these cloud-interfaces over a distance of $\sim 150 \mathrm{pc}$, one such cloud interface being that between the local cavity and the adjacent Loop I superbubble. We suggest that either (a) photo-ionization by the ambient hot gas of the Loop I cavity in addition to the stellar winds of the nearby Sco-Cen OB stars, or (b) non-equilibrium processes involving delayed recombination and fast adiabatic expansion, best explain the high ionization absorption line-components we have observed.

Our UV/visible observations have revealed at least 6 absorbing gas clouds with distances ranging from $5 \mathrm{pc}$ to $150 \mathrm{pc}$ along the 4 sight-lines. One of these clouds we believe to be the boundary to the LB cavity, which is represented by gas with a hydrogen column density $\log N(\mathrm{HI}+\mathrm{HII}) \sim 19.3 \mathrm{~cm}^{-2}$ and a distance of $\sim 90 \mathrm{pc}$ moving with a velocity of $V \sim-15 \mathrm{~km} \mathrm{~s}^{-1}$. Additionally, we have also detected the far neutral boundary to the Loop I superbubble cavity at a distance of $150-180 \mathrm{pc}$ that is composed of two clouds moving with velocities close to $V \sim 0 \mathrm{~km} \mathrm{~s}^{-1}$. This boundary seems to represent a transition region between gas with negative velocities that flows towards the local cavity and gas with positive velocities that is moving away from Loop I towards the direction of the galactic center. It is also the source of high ion absorption components of CIV and NV that are formed with a column density ratio of $N(\mathrm{CIV}) / N(\mathrm{NV}) \sim 7.3$.

Three low density, warm and partially ionized diffuse clouds with distances $<150 \mathrm{pc}$ and velocities of $\sim-10,-23$ and $-32 \mathrm{~km} \mathrm{~s}^{-1}$ have also been revealed by our observations. Velocity arguments favor the absorption observed at $V \sim$ $-23 \mathrm{~km} \mathrm{~s}^{-1}$ being associated with the very local "G-cloud" $(d<5 \mathrm{pc})$, but other factors favor its placement at a greater distance. We note that current models of the Local Bubble region predict a general flow of neutral and partially ionized clouds that are formed at a distance of 50-100 pc in the region where the neutral boundary to the local cavity and the expanding gas of the Loop I superbubble interact and then fragment into diffuse cloudlets through Rayleigh-Taylor instabilities. Such cloud fragments could possess velocities similar to that of gas in the very local interstellar medium $(d<10 \mathrm{pc})$ and thus at the present spectral resolution of our UV data would be observed as unresolved blends. However, until further high resolution absorption studies of many more sight-lines towards Loop I become available, we cannot place a firm distance limit or origin to both the $V \sim-21 \mathrm{~km} \mathrm{~s}^{-1}$ cloud, and an additional cloud component observed at $V \sim-32 \mathrm{~km} \mathrm{~s}^{-1}$.

Finally, we speculate on the relevance of our present results to understanding the physical state of highly ionized gas in the local ISM with respect to current production mechanisms. We argue that only by obtaining many more high resolution UV absorption spectra of various sight-lines within the Local Bubble can we begin to attempt to understand these processes in any real detail.

Acknowledgements. We are grateful to John Raymond, Chris McKee, Mike Shull and Ed Jenkins for their helpful comments on this paper. We would also like to recognize members of the HST project team at the Space Telescope Science Institute in Baltimore, MD. Financial support for both BYW came from Guest Observer funding from the NASA HST project under contract HST-GO-09876.02-A. BYW also recognizes partial funding from CNES (France) as part of a one-month travel grant to Verrières-le-Buisson administered by 1'Université de Versailles Saint Quentin-en-Yvelines.

\section{References}

Berkhuijsen, E., Haslam, C., \& Salter, C. 1971, A\&A, 14, 252

Bertin, P., Vidal-Madjar, A., Lalelment, R., Ferlet, R., \& Lemoine, M. 1995, A\&A, 302, 889

Bohringer, H., \& Hartquist, T. 1987, MNRAS, 228, 915

Borkowski, K. J., Sarazin, C. L., \& Soker, N. 1990, ApJ, 360, 173

Breitschwerdt, D. 2001, Ap\&SS, 276, 163

Breitschwerdt, D., \& Schmutzler, T. 1994, Nature, 371, 774

Breitschwerdt, D., Freyberg, M., \& Egger, R. 2000, A\&A, 361, 303

Centurion, M., \& Vladilo, G. 1991, ApJ, 372, 494

Corradi, W., Franco, G., \& Knude, J. 2004, MNRAS, 347, 1065

Cox, D. P., \& Helenius, L. 2003, ApJ, 583, 205

Cravens, T. E., Robertson, I. E., \& Snowden, S. 2001, JGR, 106, 24883

Crawford, I. 1991, A\&A, 247, 183

Crawford, I. 1992, MNRAS, 254, 264

Crawford, I. 2000, MNRAS, 317, 996

Crawford, I. 2001, MNRAS, 327, 841

Crawford, I., \& Dunkin, S. 1995, MNRAS, 273, 219 
Crawford, I., Craig, N., \& Welsh, B. Y. 1997, A\&A, 317, 889

Crawford, I., Lallement, R., Price, R. J., et al. 2002, MNRAS, 337, 720

de Geus, E., de Zeeuw, P., \& Lub, J. 1989, A\&A, 216, 44

de Geus, E. 1992, A\&A, 262, 258

Edgar, R., \& Chevalier, R. 1986, ApJ, 310, L27

Egger, R., \& Aschenbach, B. 1995, A\&A, 294, L25

ESA 1997, The Hipparcos Satellite Catalogue, ESA SP-1200

Ferlet, R., Vidal-Madjar, A., \& Gry, C. 1985, ApJ, 298, 838

Fox, A., Savage, B. D., Sembach, K., et al. 2003, ApJ, 582, 793

Gry, C., \& Jenkins, E. 2001, A\&A, 367, 617

Heiles, C. 1998, ApJ, 498, 689

Holberg, J., Bruhweiler, F., Barstow, M., \& Dobbie, P. 1999, ApJ, 517, 841

Howk, J. C., \& Savage, B. 1999, ApJ, 517, 746

Huang, J.-S., Songaila, A., Cowie, L., \& Jenkins, E. 1995, ApJ, 450, 163

Hurwitz, M., Sasseen, T., \& Sirk, M. 2005, ApJ, in press

Indebetouw, R., \& Shull, M. 2004, ApJ, 605, 205

Iwan, D. 1980, ApJ, 239, 316

Jelinsky, P., Vallerga, J. V., \& Edelstein, J. 1995, ApJ, 442, 653

Jenkins, E., \& Shaya, E. 1979, ApJ, 231, 55

Knauth, D., Howk, J., Sembach, K., Lauroesch, J., \& Meyer, D. 2003, ApJ, 592, 964

Lallement, R., \& Bertin, P. 1992, A\&A, 266, 479

Lallement, R., Ferlet, R., Lagrange, A. M., Lemoine, M., \& Vidal-Madjar, A. 1995, A\&A, 304, 461

Lalelment, R., Welsh, B. Y., Vergerly, J. L., Crifo, F., \& Sfeir, D. 2003, A\&A, 411, 447

Lallement, R. 2004, A\&A, 418, 143

Lehner, N., Jenkins, E., Gry, C., et al. 2003, ApJ, 595, 858

Linsky, J., \& Wood, B. 1996, ApJ, 463, 254

Lyu, C.-H., \& Bruhweiler, F. 1996, ApJ, 459, 216

Morton, D. C. 2003, ApJS, 149, 205

Oegerle, W., Jenkins, E. B., Shelton, R., Bowen, D., \& Chayer, P. 2005, ApJ, 622, 377
Pan, K., Federman, S., Cunha, K., Smith, V., \& Welty, D. 2004, ApJS, 151,313

Redfield, S., \& Linsky, J. 2002, ApJS, 139, 439

Savage, B., \& Sembach, K. 1996, ARA\&A, 34, 279

Savage, B., Meade, M., \& Sembach, K. 2001, ApJS, 136, 631

Sembach, K., \& Savage, B. 1992, ApJS, 83, 147

Sembach, K., \& Savage, B. 1996, ApJ, 457, 211

Sembach, K., Savage, B., \& Tripp, T. 1997, ApJ, 480, 216

Sfeir, D. 1999, Thesis, University of Paris

Sfeir, D., Lallement, R., Crifo, F., \& Welsh, B. Y. 1999, A\&A, 346, 785

Shelton, R. 2003, ApJ, 589, 261

Slavin, J. D. 1989, ApJ, 346, 718

Slavin, J., \& Frisch, P. 2002, ApJ, 565, 364

Smith, R. K., et al. 2005, ApJ, submitted

Snowden, S., Egger, R., Finkbeiner, D. P., Freyberg, M. J., \& Plucinsky, P. P. 1998, ApJ, 493, 715

Sofue, Y., Hamajima, K., \& Fujimoto, M. 1974, PASJ, 26, 399

Vallerga, J. V., Vedder, P., Craig, N., \& Welsh, B. Y. 1993, ApJ, 411, 729

Vallerga, J. V., \& Welsh, B. Y. 1995, ApJ, 444, 702

Weaver, R., McCray, R., Castor, J., et al. 1977, ApJ, 218, 377

Welsh, B. Y., Vedder, P., \& Vallerga, J. 1990, ApJ, 358, 473

Welsh, B. Y., Craig, N., Vedder, P., \& Vallerga, J. V. 1994, ApJ, 437, 638

Welsh, B. Y., Sfeir, D., Sirk, M., \& Lallement, R. 1999, A\&A, 352, 308

Welsh, B. Y., Sallmen, S., Sfeir, D., Shelton, R., \& Lallement, R. 2002, A\&A, 394, 691

Welty, D., Hobbs, L., \& Kulkarni, V. 1994, ApJ, 436, 152

Willingdale, R., Hands, A., Warwick, R., et al. 2003, MNRAS, 343, 995

Wolff, B., Koester, D., \& Lallement, R. 1999, A\&A, 346, 969

Wood, B., Linsky, J., \& Zank, G. 2000, ApJ, 537, 304

Woodgate, B. E., Kimble, R. A., Bowers, C., et al. 1998, PASP, 110, 1183 